\title{
Search for dark matter signals towards a selection of recently detected DES dwarf galaxy satellites of the Milky Way with H.E.S.S.
}

H. Abdallah, ${ }^{1}$ R. Adam,${ }^{2}$ F. Aharonian,${ }^{3,45}$ F. Ait Benkhali, ${ }^{3}$ E. O. Angüner, ${ }^{6}$ M. Arakawa, ${ }^{7}$ C. Arcaro, ${ }^{1}$ C. Armand,${ }^{8, *}$ T. Armstrong, ${ }^{9}$ H. Ashkar ${ }^{10}$ M. Backes, ${ }^{11}, 1$ V. Baghmanyan, ${ }^{12}$ V. Barbosa Martins,${ }^{13}$ A. Barnacka ${ }^{14}$ M. Barnard,${ }^{1}$ Y. Becherini, ${ }^{15}$ D. Berge,${ }^{13}$ K. Bernlöhr, ${ }^{3}$ M. Böttcher, ${ }^{1}$ C. Boisson, ${ }^{16}$ J. Bolmont,${ }^{17}$ S. Bonnefoy, ${ }^{13}$ M. Breuhaus, ${ }^{3}$ J. Bregeon, ${ }^{18}$ F. Brun,${ }^{10}$ P. Brun,${ }^{10}$ M. Bryan, ${ }^{19}$ M. Büchele ${ }^{20}$ T. Bulik, ${ }^{21}$ T. Bylund, ${ }^{15}$ S. Caroff, ${ }^{17}$ A. Carosi,${ }^{8}$ S. Casanova, ${ }^{12,3}$ T. Chand, ${ }^{1}$ S. Chandra, ${ }^{1}$ A. Chen,${ }^{22}$ G. Cotter,${ }^{9}$ M. Curyło, ${ }^{21}$ I. D. Davids, ${ }^{11}$ J. Davies, ${ }^{9}$ C. Deil, ${ }^{3}$ J. Devin ${ }^{23}$ P. deWilt, ${ }^{24}$ L. Dirson, ${ }^{25}$ A. Djannati-Ataï, ${ }^{26}$ A. Dmytriiev, ${ }^{16}$ A. Donath, ${ }^{3}$ V. Doroshenko, ${ }^{27}$ J. Dyks,${ }^{28} \mathrm{~K}$. Egberts, ${ }^{29}$ F. Eichhorn, ${ }^{20}$ G. Emery ${ }^{17}$ J.-P. Ernenwein, ${ }^{6}$ S. Eschbach ${ }^{20}{ }^{20}$ K. Feijen, ${ }^{24}$ S. Fegan, ${ }^{2}$ A. Fiasson, ${ }^{8}$ G. Fontaine, ${ }^{2}$ S. Funk ${ }^{20}$ M. Füßling, ${ }^{13}$ S. Gabici, ${ }^{26}$ Y. A. Gallant,${ }^{13}$ G. Giavitto, ${ }^{13}$ L. Giunti, ${ }^{26}$ D. Glawion, ${ }^{30}$ J. F. Glicenstein, ${ }^{10}$ D. Gottschall, ${ }^{27}$ M.-H. Grondin, ${ }^{23}$ J. Hahn, ${ }^{3}$ M. Haupt, ${ }^{13}$ G. Hermann, ${ }^{3}$ J. A. Hinton, ${ }^{3}$ W. Hofmann, ${ }^{3}$ C. Hoischen, ${ }^{29}$ T. L. Holch, ${ }^{31}$

M. Holler, ${ }^{32}$ M. Hörbe, ${ }^{9}$ D. Horns,${ }^{25}$ D. Huber, ${ }^{32}$ H. Iwasaki, ${ }^{7}$ M. Jamrozy,${ }^{33}$ D. Jankowsky, ${ }^{20}$ F. Jankowsky, ${ }^{30}$

A. Jardin-Blicq, ${ }^{3}$ V. Joshi, ${ }^{20}$ I. Jung-Richardt, ${ }^{20}$ M. A. Kastendieck, ${ }^{25}$ K. Katarzyński, ${ }^{34}$ M. Katsuragawa, ${ }^{35}$ U. Katz ${ }^{20}$ D. Khangulyan, ${ }^{7}$ B. Khélifi, ${ }^{26}$ S. Klepser, ${ }^{13}$ W. Kluźniak, ${ }^{28}$ Nu. Komin, ${ }^{22}$ R. Konno, ${ }^{13}$ K. Kosack, ${ }^{10}$ D. Kostunin, ${ }^{13}$ M. Kreter, ${ }^{1}$ G. Lamanna, ${ }^{8}$ A. Lemière, ${ }^{26}$ M. Lemoine-Goumard, ${ }^{23}$ J.-P. Lenain, ${ }^{17}$ E. Leser, ${ }^{29,13}$ C. Levy, ${ }^{17}$ T. Lohse, ${ }^{31}$ I. Lypova, ${ }^{13}$ J. Mackey, ${ }^{4}$ J. Majumdar, ${ }^{13}$ D. Malyshev, ${ }^{27}$ D. Malyshev, ${ }^{20}$ V. Marandon, ${ }^{3}$ P. Marchegiani, ${ }^{22}$ A. Marcowith, ${ }^{18}$ A. Mares,${ }^{23}$ G. Martì-Devesa ${ }^{32}$ R. Marx,${ }^{30,3}$ G. Maurin, ${ }^{8}$ P. J. Meintjes, ${ }^{36}$ R. Moderski, ${ }^{28}$ M. Mohamed,${ }^{30}$ L. Mohrmann, ${ }^{20}$ C. Moore ${ }^{37}$ P. Morris, ${ }^{9}$ E. Moulin $\odot,{ }^{10,}{ }^{*}$ J. Muller, ${ }^{2}$ T. Murach,${ }^{13}$ K. Nakashima,${ }^{20}$ S. Nakashima ${ }^{38}$ M. de Naurois, ${ }^{2}$ H. Ndiyavala, ${ }^{1}$ F. Niederwanger, ${ }^{32}$ J. Niemiec, ${ }^{12}$ L. Oakes, ${ }^{31}$ P. O'Brien ${ }^{37}$ H. Odaka, ${ }^{39}$ S. Ohm, ${ }^{13}$ E. de Ona Wilhelmi, ${ }^{13}$

M. Ostrowski, ${ }^{33}$ M. Panter, ${ }^{3}$ R. D. Parsons, ${ }^{3}$ B. Peyaud,${ }^{10}$ Q. Piel,${ }^{8}$ S. Pita,${ }^{26}$ V. Poireau, ${ }^{8,}$ A. Priyana Noel, ${ }^{14}$

D. A. Prokhorov ${ }^{22,15}$ H. Prokoph, ${ }^{13}$ G. Pühlhofer, ${ }^{27}$ M. Punch,${ }^{26,15}$ A. Quirrenbach, ${ }^{30}$ S. Raab, ${ }^{20}$ R. Rauth, ${ }^{32}$ A. Reimer, ${ }^{32}$ O. Reimer, ${ }^{32}$ Q. Remy, ${ }^{3}$ M. Renaud ${ }^{18}$ F. Rieger, ${ }^{3}$ L. Rinchiuso $\odot,{ }^{10, *}$ C. Romoli, ${ }^{3}$ G. Rowell, ${ }^{24}$ B. Rudak, ${ }^{28}$

E. Ruiz-Velasco, ${ }^{3}$ V. Sahakian, ${ }^{40}$ S. Sailer, ${ }^{3}$ S. Saito, ${ }^{7}$ D. A. Sanchez, ${ }^{8}$ A. Santangelo, ${ }^{27}$ M. Sasaki,${ }^{20}$ M. Scalici, ${ }^{27}$ F. Schüssler ${ }^{10}$ H. M. Schutter, ${ }^{1}$ U. Schwanke, ${ }^{31}$ S. Schwemmer, ${ }^{30}$ M. Seglar-Arroyo, ${ }^{10}$ M. Senniappan, ${ }^{15}$ A. S. Seyffert, ${ }^{1}$ N. Shafi, ${ }^{22}$ K. Shiningayamwe, ${ }^{11}$ R. Simoni ${ }^{19}$ A. Sinha,${ }^{26}$ H. Sol, ${ }^{16}$ A. Specovius, ${ }^{20}$ S. Spencer, ${ }^{9}$ M. Spir-Jacob,${ }^{26}$ Ł. Stawarz, ${ }^{33}$ R. Steenkamp, ${ }^{11}$ C. Stegmann, ${ }^{29,13}$ C. Steppa, ${ }^{29}$ T. Takahashi, ${ }^{35}$ T. Tavernier, ${ }^{10}$ A. M. Taylor, ${ }^{13}$ R. Terrier, ${ }^{26}$ D. Tiziani, ${ }^{20}$ M. Tluczykont, ${ }^{25}$ L. Tomankova, ${ }^{20} \mathrm{C}$. Trichard, ${ }^{2} \mathrm{M}$. Tsirou, ${ }^{18} \mathrm{~N}$. Tsuji, ${ }^{7}$ R. Tuffs, ${ }^{3}$ Y. Uchiyama, ${ }^{7}$ D. J. van der Walt, ${ }^{1}$ C. van Eldik, ${ }^{20}$ C. van Rensburg, ${ }^{1}$ B. van Soelen, ${ }^{36}$ G. Vasileiadis,${ }^{18}$ J. Veh,${ }^{20}$ C. Venter, ${ }^{1}$ A. Viana, ${ }^{41}$ P. Vincent, ${ }^{17}$ J. Vink, ${ }^{19}$ H. J. Völk, ${ }^{3}$ T. Vuillaume, ${ }^{8}$ Z. Wadiasingh, ${ }^{1}$ S. J. Wagner, ${ }^{30}$ J. Watson, ${ }^{9}$ F. Werner, ${ }^{3}$ R. White, ${ }^{3}$ A. Wierzcholska, ${ }^{12,30}$ R. Yang, ${ }^{3}$ H. Yoneda, ${ }^{35}$ M. Zacharias, ${ }^{1}$ R. Zanin, ${ }^{3}$ D. Zargaryan, ${ }^{4}$ A. A. Zdziarski, ${ }^{28}$ A. Zech, ${ }^{16}$ S. Zhu, ${ }^{13}$ J. Zorn, ${ }^{3}$ and N. Żywucka ${ }^{1}$

\section{(H.E.S.S. Collaboration)}

\author{
${ }^{1}$ Centre for Space Research, North-West University, Potchefstroom 2520, South Africa \\ ${ }^{2}$ Laboratoire Leprince-Ringuet, cole Polytechnique, CNRS, Institut Polytechnique de Paris, \\ F-91128 Palaiseau, France \\ ${ }^{3}$ Max-Planck-Institut für Kernphysik, P.O. Box 103980, D 69029 Heidelberg, Germany \\ ${ }^{4}$ Dublin Institute for Advanced Studies, 31 Fitzwilliam Place, Dublin 2, Ireland \\ ${ }^{5}$ High Energy Astrophysics Laboratory, RAU, 123 Hovsep Emin Street, Yerevan 0051, Armenia \\ ${ }^{6}$ Aix Marseille Université, CNRS/IN2P3, CPPM, 13288 Marseille, France \\ ${ }^{7}$ Department of Physics, Rikkyo University, 3-34-1 Nishi-Ikebukuro, Toshima-ku, Tokyo 171-8501, Japan \\ ${ }^{8}$ Laboratoire d'Annecy de Physique des Particules, Université Grenoble Alpes, \\ Université Savoie Mont Blanc, CNRS, LAPP, 74000 Annecy, France \\ ${ }^{9}$ University of Oxford, Department of Physics, Denys Wilkinson Building, \\ Keble Road, Oxford OX1 3RH, United Kingdom \\ ${ }^{10}$ IRFU, CEA, Université Paris-Saclay, F-91191 Gif-sur-Yvette, France \\ ${ }^{11}$ University of Namibia, Department of Physics, Private Bag 13301 Windhoek, Namibia \\ ${ }^{12}$ Instytut Fizyki Jądrowej PAN, ul. Radzikowskiego 152, 31-342 Kraków, Poland \\ ${ }^{13}$ DESY, D-15738 Zeuthen, Germany \\ ${ }^{14}$ Obserwatorium Astronomiczne, Uniwersytet Jagiellonski, ul. Orla 171, 30-244 Kraków, Poland \\ ${ }^{15}$ Department of Physics and Electrical Engineering, Linnaeus University, 35195 Växjö, Sweden \\ ${ }^{16}$ LUTH, Observatoire de Paris, PSL Research University, CNRS, Université Paris Diderot, \\ 5 Place Jules Janssen, 92190 Meudon, France
}




\author{
${ }^{17}$ Sorbonne Université, Université Paris Diderot, Sorbonne Paris Cité, CNRS/IN2P3, \\ Laboratoire de Physique Nucléaire et de Hautes Energies, \\ LPNHE, 4 Place Jussieu, F-75252 Paris, France \\ ${ }^{18}$ Laboratoire Univers et Particules de Montpellier, Université Montpellier, CNRS/IN2P3, \\ CC 72, Place Eugène Bataillon, F-34095 Montpellier Cedex 5, France \\ ${ }^{19}$ GRAPPA, Anton Pannekoek Institute for Astronomy and Institute of High-Energy Physics, \\ University of Amsterdam, Science Park 904, 1098 XH Amsterdam, The Netherlands \\ ${ }^{20}$ Friedrich-Alexander-Universität Erlangen-Nürnberg, Erlangen Centre for Astroparticle Physics, \\ Erwin-Rommel-Straße 1, D 91058 Erlangen, Germany \\ ${ }^{21}$ Astronomical Observatory, The University of Warsaw, Al. Ujazdowskie 4, 00-478 Warsaw, Poland \\ ${ }^{22}$ School of Physics, University of the Witwatersrand, \\ 1 Jan Smuts Avenue, Braamfontein, Johannesburg 2050, South Africa \\ ${ }^{23}$ Université Bordeaux, CNRS/IN2P3, Centre d'Études Nucléaires de Bordeaux Gradignan, \\ 33175 Gradignan, France \\ ${ }^{24}$ School of Physical Sciences, University of Adelaide, Adelaide 5005, Australia \\ ${ }^{25}$ Universität Hamburg, Institut für Experimentalphysik, \\ Luruper Chaussee 149, D 22761 Hamburg, Germany \\ ${ }^{26}$ APC, AstroParticule et Cosmologie, Université Paris Diderot, CNRS/IN2P3, CEA/Irfu, \\ Observatoire de Paris, Sorbonne Paris Cité, 10, rue Alice Domon et Léonie Duquet, \\ 75205 Paris Cedex 13, France \\ ${ }^{27}$ Institut für Astronomie und Astrophysik, Universität Tübingen, Sand 1, D 72076 Tübingen, Germany \\ ${ }^{28}$ Nicolaus Copernicus Astronomical Center, Polish Academy of Sciences, \\ ul. Bartycka 18, 00-716 Warsaw, Poland \\ ${ }^{29}$ Institut für Physik und Astronomie, Universität Potsdam, \\ Karl-Liebknecht-Strasse 24/25, D 14476 Potsdam, Germany \\ ${ }^{30}$ Landessternwarte, Universität Heidelberg, Königstuhl, D 69117 Heidelberg, Germany \\ ${ }^{31}$ Institut für Physik, Humboldt-Universität zu Berlin, Newtonstraße 15, D 12489 Berlin, Germany \\ ${ }^{32}$ Institut für Astro- und Teilchenphysik, Leopold-Franzens-Universität Innsbruck, \\ A-6020 Innsbruck, Austria \\ ${ }^{33}$ Obserwatorium Astronomiczne, Uniwersytet Jagielloński, ul. Orla 171, 30-244 Kraków, Poland \\ ${ }^{34}$ Centre for Astronomy, Faculty of Physics, Astronomy and Informatics, Nicolaus Copernicus University, \\ Grudziadzka 5, 87-100 Toruń, Poland \\ ${ }^{35}$ Kavli Institute for the Physics and Mathematics of the Universe (WPI), \\ The University of Tokyo Institutes for Advanced Study (UTIAS), The University of Tokyo, \\ 5-1-5 Kashiwa-no-Ha, Kashiwa, Chiba 277-8583, Japan \\ ${ }^{36}$ Department of Physics, University of the Free State, PO Box 339, Bloemfontein 9300, South Africa \\ ${ }^{37}$ Department of Physics and Astronomy, The University of Leicester, \\ University Road, Leicester LE1 7RH, United Kingdom \\ ${ }^{38}$ RIKEN, 2-1 Hirosawa, Wako, Saitama 351-0198, Japan \\ ${ }^{39}$ Department of Physics, The University of Tokyo, 7-3-1 Hongo, Bunkyo-ku, Tokyo 113-0033, Japan \\ ${ }^{40}$ Yerevan Physics Institute, 2 Alikhanian Brothers Street, 375036 Yerevan, Armenia \\ ${ }^{41}$ Now at Instituto de Física de São Carlos, Universidade de São Paulo, \\ Avenida Trabalhador São-carlense, 400_CEP 13566-590 São Carlos, SP, Brazil
}

(Received 24 March 2020; accepted 31 July 2020; published 2 September 2020)

Dwarf spheroidal galaxy satellites of the Milky Way are prime targets for indirect detection of dark matter with gamma rays due to their proximity, high dark matter content, and absence of nonthermal emission processes. Recently, the Dark Energy Survey (DES) revealed the existence of new ultrafaint dwarf spheroidal galaxies in the southern-hemisphere sky, therefore ideally located for ground-based observations with the imaging atmospheric Cherenkov telescope array H.E.S.S. We present a search for very-highenergy $(E \gtrsim 100 \mathrm{GeV})$ gamma-ray emission using H.E.S.S. observations carried out recently towards Reticulum II, Tucana II, Tucana III, Tucana IV, and Grus II satellites. No significant very-high-energy gamma-ray excess is found from the observations on any individual object nor in the combined analysis of all the datasets. Using the most recent modeling of the dark matter distribution in the dwarf galaxy halo, we compute for the first time on DES satellites individual and combined constraints from Cherenkov telescope

\footnotetext{
*Corresponding authors.contact. hess@hess-experiment.eu
} 
observations on the annihilation cross section of dark matter particles in the form of Weakly Interacting Massive Particles. The combined 95\% C.L. observed upper limits reach $\langle\sigma v\rangle \simeq 1 \times 10^{-23} \mathrm{~cm}^{3} \mathrm{~s}^{-1}$ in the $W^{+} W^{-}$channel and $4 \times 10^{-26} \mathrm{~cm}^{3} \mathrm{~s}^{-1}$ in the $\gamma \gamma$ channels for a dark matter mass of $1.5 \mathrm{TeV}$. The H.E.S.S. constraints well complement the results from Fermi-LAT, HAWC, MAGIC, and VERITAS and are currently the most stringent in the $\gamma \gamma$ channels in the multi-GeV/multi-TeV mass range.

DOI: 10.1103/PhysRevD.102.062001

\section{INTRODUCTION}

Precise cosmological measurements [1] support the theory that most of the matter in the Universe is composed of nonbaryonic cold dark matter (DM). The search for nongravitational interactions of DM is one of the major efforts in contemporary fundamental astrophysics. Despite the worldwide multifaceted efforts that have been deployed over the last decades to detect DM, its nature is presently unknown. Many theoretical models [2] have been devised to propose DM particle candidates. Among them is a Weakly Interacting Massive Particle (WIMP) with mass and coupling at the electroweak scale that provides the cold DM density measured in the Universe today [3], which is popularly acknowledged as the WIMP miracle. Among the experimental strategies devised to detect DM, the indirect searches look for the Standard Model particles produced during the DM annihilation or decay. WIMPs could still annihilate today in dense regions of the Universe producing very-high-energy (VHE, $\mathrm{E} \gtrsim 100 \mathrm{GeV}$ ) gamma rays in the final states that can be eventually detected by ground-based imaging atmospheric Cherenkov telescopes (IACT) such as the High Energy Stereoscopic System (H.E.S.S.).

Among the most favorable environments to look for DM annihilation in VHE gamma rays are dwarf spheroidal galaxies (dSphs) satellites of the Milky Way, with many of them nearby and at high Galactic latitudes. The measured stellar kinematics in dSphs make them the most DMdominated objects in the Universe. They are composed of old stellar populations and contain little gas which could act as target materials for VHE cosmic rays. No hint is found for nonthermal processes that could give rise to emission from non-DM scenarios which would serve as background for a DM search in VHE gamma rays [4,5]. Despite the lower DM signals expected for dSphs compared to the central region of the Milky Way, they have the advantage of negligible background emission to hide a DM signal.

Numerous dSphs have been discovered via the Sloan Digital Sky Survey [6] covering the Northern celestial hemisphere. More recently ultrafaint $\mathrm{dSphs}$ are being unveiled by the ongoing surveys like PanSTARRS [7], and the DES [8], with the prospect of more discoveries with the Large Synoptic Survey Telescope [9]. DES is a southern-hemisphere optical survey providing photometric measurements to detect stellar overdensities with unprecedented sensitivity in the southern sky. The ultrafaint
Milky Way satellites newly discovered by DES are consistent with being dSphs while a fraction of them are referred to as dSph candidates in absence of confirmation from spectroscopic measurements. They represent new promising targets for VHE gamma-ray searches for DM annihilations.

We present here the observations carried out by H.E.S.S. on a selection of DES satellites to search for DM annihilation signals. The targeted systems are Reticulum II (Ret II), Tucana II (Tuc II), Tucana III (Tuc III), and Grus II (Gru II), with Tucana IV (Tuc IV) in the field of view (FoV) of Tuc III observations. The results of the search for DM annihilation signals are presented for individual and combined searches towards these targets. The paper is organized as follows. Section II presents the DM signals expected from the targets. In Secs. III and IV, we present the observational datasets, and the data analysis, respectively. Section V is devoted to the results. We conclude in Sec. VI.

\section{DARK MATTER SIGNALS}

\section{A. Dark matter distribution and gamma-ray flux}

The energy-differential gamma-ray flux expected from the self-annihilation of DM particles of mass $m_{\mathrm{DM}}$ in the region of solid angle $\Delta \Omega$ can be written as [2]:

$$
\begin{aligned}
\frac{\mathrm{d} \Phi_{\gamma}}{\mathrm{d} E_{\gamma}}\left(E_{\gamma}, \Delta \Omega\right) & =\frac{\langle\sigma v\rangle}{8 \pi m_{\mathrm{DM}}^{2}} \sum_{\mathrm{f}} \mathrm{BR}_{\mathrm{f}} \frac{\mathrm{d} N^{\mathrm{f}}}{\mathrm{d} E_{\gamma}} J(\Delta \Omega), \quad \text { with } \\
J(\Delta \Omega) & =\int_{\Delta \Omega} \int_{\mathrm{LoS}} \rho^{2}(s(r, \theta)) d s d \Omega .
\end{aligned}
$$

The first term groups the total velocity-weighted annihilation cross section $\langle\sigma v\rangle$ and the sum of the annihilation spectra $d N^{f} / d E_{\gamma}$ in the final states $f$ with associated branching ratios $\mathrm{BR}_{f}$. The second term, often referred to as the $J$-factor, corresponds to the square of the DM mass density $\rho$ integrated over the line-of-sight (LoS) $s$ and $\Delta \Omega$. The distance from the observer to the annihilation location $s$ is given by $r=\left(s^{2}+s_{0}^{2}-2 s_{0} s \cos \theta\right)^{1 / 2}$, where $s_{0}$ is the distance from the target to the Earth and $\theta$ the angle between the direction of observation and the $\mathrm{dSph}$ center. The DM mass density is inferred from the measurements of the position and LoS velocity of the stars gravitationally bound in the dwarf galaxy potential well through the Jeans 
equation [10]. The finite number of kinematic measurements of the member stars leads to an uncertainty on the $J$-factor; see, e.g., Ref. [11].

The expected DM flux is composed of a continuum spectrum extending up to the DM mass, and a line emission feature. The former contribution arises from the hadronization and/or decay of quarks, heavy leptons, and gauge bosons involved in the annihilation process. The latter comes from the prompt annihilation into $\gamma X$ with $X=\gamma, \mathrm{h}, \mathrm{Z}$, or a nonstandard model neutral particle, providing a spectral line at an energy $E_{\gamma}=$ $m_{\mathrm{DM}}\left[1-\left(m_{X} / 2 m_{\mathrm{DM}}\right)^{2}\right]$. Additional gamma rays are produced when the DM particles self-annihilate into charged particles via processes involving virtual internal bremsstrahlung and final state radiation. Such processes provide a wider linelike feature that peaks at an energy close to $m_{\mathrm{DM}}$.

\section{B. Target selection}

Five targets were selected among the 16 newly discovered DES dSphs [12,13]. The selection is based mainly on the DM content and visibility from the H.E.S.S. site. Targets with measured or predicted $J$-factor close to $\log _{10}\left(J_{<0.5^{\circ}} / \mathrm{GeV}^{2} \mathrm{~cm}^{-2}\right) \sim 19$ are chosen, with visibility at zenith angles lower than $60^{\circ}$ spread all over the year. The priority has been given to targets that are confirmed as dwarf galaxies, followed by the galaxy candidates with the largest $J$-factor. The chosen targets are outlined in Table I. The low-luminosity Milky Way satellite Ret II has been discovered using photometric data from the DES $[12,14]$. Located at a distance of $32 \mathrm{kpc}$ from the Sun, it is one of the nearest dSphs after Segue $1(23 \mathrm{kpc})$ [15] and Sagittarius (24 kpc) [16]. Ret II is about three times more luminous than Segue 1, which suggests that its DM halo may be more massive than that of Segue 1. This makes it a privileged $\mathrm{dSph}$ target for $\mathrm{DM}$ searches. Assuming dynamical equilibrium and spherical symmetry, a Jeans analysis of the available kinematic data suggest that the $J$-factor of Ret II is among the highest of the known dSphs. Its $J$-factor integrated within a radius of $0.5^{\circ}$ reaches $\log _{10}\left(J / \mathrm{GeV}^{2} \mathrm{~cm}^{-5}\right)=19.6$ [17] based on a kinematic sample of 38 member stars. Alternative studies derived mean $\log _{10}\left(\mathrm{~J} / \mathrm{GeV}^{2} \mathrm{~cm}^{-5}\right)$ values as large as 20.5 [18] and as low as 18.2 [19] within a $0.5^{\circ}$ radius. A systematic study presented in Refs. [17,20] shows that its $J$-factor determination is stable against assumptions in the Jeans analysis. No hints of tidal disruption [21] or a significant binary star population [22] in Ret II have been detected so far. Based on the velocities and metallicities of its stars, Ret II is confirmed as an ultrafaint dwarf galaxy [23]. Present photometric and spectroscopic data cannot constrain the fraction of binary stars in the kinematic stellar sample. In the observed absence of tidal disruption and binary stars that would artificially inflate the velocity dispersion, Ret II is a prime DM target for H.E.S.S.

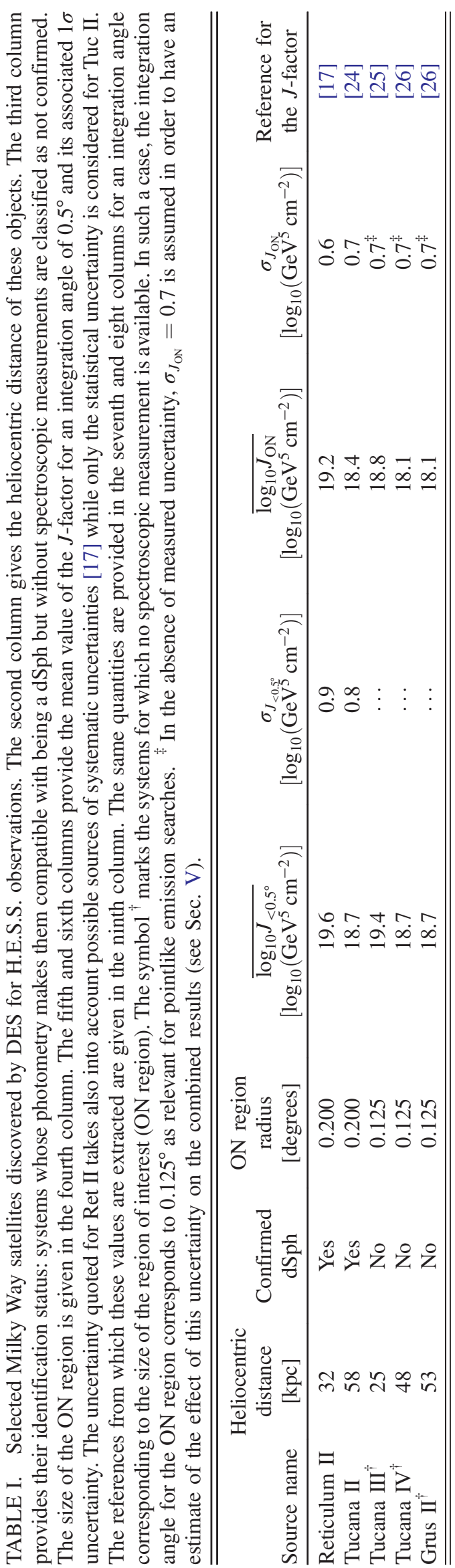


Tuc II is an ultrafaint dSph galaxy Milky Way satellite discovered from DES photometric data [12] located at $57 \mathrm{kpc}$ from the Sun. Spectroscopic measurements of member stars [24] reveal a low internal velocity dispersion. Assuming dynamical equilibrium, spherical symmetry, and a negligible contamination of binary stars in the stellar sample, the $J$-factor of Tuc II is calculated as $\log _{10}\left(\mathrm{~J} / \mathrm{GeV}^{2} \mathrm{~cm}^{-5}\right)=18.7$ within $0.5^{\circ}$ [24], which makes it an interesting DM target among known dSphs. Spectroscopic observations of member stars of Tuc II classify it as a dwarf galaxy [24]. No tidal disruption or significant binary star population have been measured so far in this system. In Ref. [27] the $J$-factor of Tuc II is predicted to be $\log _{10}\left(\mathrm{~J} / \mathrm{GeV}^{2} \mathrm{~cm}^{-5}\right)=19.0$ within a $0.5^{\circ}$ radius [27]. The conservative estimate is used in what follows.

Among the dSph candidates discovered by DES [12], Tuc III is the nearest low-luminosity Milky Way satellite located at a heliocentric distance of $25 \mathrm{kpc}$ [25]. Spectroscopic measurements show a very low velocity dispersion in the member stars, and only upper limits can be safely derived. Despite its larger size and lower surface brightness compared to any known globular cluster, Tuc III cannot be confirmed as a DM-dominated system, and, therefore, cannot be definitely classified as a dSph. If Tuc III has a DM halo similar to the one of other satellites with similar stellar mass, the $J$-factor of Tuc III can be as high as $\log _{10}\left(\mathrm{~J} / \mathrm{GeV}^{2} \mathrm{~cm}^{-5}\right)=19.4$ within $0.5^{\circ}$ [25] making it a very promising DM target that can be conveniently observed by H.E.S.S. However, the derivation of the $J$-factor from the modeling of the velocity distribution suffers from systematic uncertainties and $J$-factors as low as $\log _{10}\left(J / \mathrm{GeV}^{2} \mathrm{~cm}^{-5}\right) \simeq 17.8$ are possible [25]. The predicted values from Ref. [26] and Ref. [27] are $\log _{10}\left(\mathrm{~J} / \mathrm{GeV}^{2} \mathrm{~cm}^{-5}\right)=19.0$ and 17.7 , respectively, within $0.5^{\circ}$.

No accurate spectroscopic measurements are available for Gru II and Tuc IV. In absence of measurements of the velocity dispersion of member stars, they are classified as likely dSphs [26]. No $J$-factor can be measured and the empirical law from Ref. [26] is used to provide an estimate of the $J$-factor of Gru II and Tuc IV.

The determination of the DM density distribution in $\mathrm{dSphs}$ is subject to uncertainties that can significantly affect the $J$-factor estimation. Due to the finite sample of stellar tracers in dSphs, the statistical uncertainty on the $J$-factor is higher for ultrafaint candidates than that of the classical dSphs such as Sculptor or Draco. The Jeans equation framework assumes dynamical equilibrium of stellar tracers (e.g., no tidal disruption), spherical symmetry of the system, light profile parametrization, and radial dependence of the velocity anisotropy. This set of hypotheses may not be valid in the physical systems, which would lead to systematic uncertainties in the $J$-factor determination. In what follows, the statistical uncertainties for the computation of the limits are considered when the spectroscopic measurements are available. Sources of systematic uncertainties in the $J$-factor determination in dSphs are discussed, for instance, in Refs. [28,29].

The ultrafaint systems Tuc III, Tuc IV, and Gru II lack spectroscopic measurements of their member stars and their $J$-factor can only be predicted. Assuming these objects to be embedded in spherical cuspy DM halo following the relationship between their enclosed mass, velocity dispersion, and half-light radius, an analytic formula to calculate the $J$-factors can be derived [19]. An alternative method based on a distance scaling relationship provides a compatible estimate of the $J$-factor assuming that the stellar systems lie in DM halos similar to those of known dSphs as shown in Ref. [26]. For Tuc III, the $J$-factor value within $0.5^{\circ}$ can be as large as $\log _{10} J=19.4$ using the lower limit on its mass from the tidal stripping argument [25]. However, the derived value from an upper limit on the LoS velocity dispersion is about two orders of magnitude below. Recent photometric observations classify Tuc III as an ultrafaint dwarf galaxy [23] while spectroscopic measurements failed so far to unambiguously confirm its dynamical status [25]. In absence of lower limits on the velocity dispersion for Tuc IV and Gru II, the $J$-factor values in the region of interest (ROI) are derived following the methodology of Ref. [26] assuming an inner slope of one for a cuspy DM profile [19]. In the case of Tuc III, Tuc IV, and Gru II, no statistical uncertainty can be derived for their $J$-factors. $\sigma_{J_{\mathrm{ON}}}=0.7$ is assumed to have an estimate of the degradation of the H.E.S.S. limits towards these objects when considering the $J$-factor uncertainty. Such a value is coherent given the measured uncertainty derived for Ret II and Tuc II.

\section{OBSERVATIONS AND DATASET}

H.E.S.S. is an array of five IACTs situated in the Khomas Highland in Namibia, at $1800 \mathrm{~m}$ above the sea level. The observatory consists of four $12 \mathrm{~m}$ diameter telescopes (CT1-4) at the corner of a square of side length $120 \mathrm{~m}$ and a fifth $28 \mathrm{~m}$ diameter telescope (CT5) at the middle of the array since 2012. Given its location in the Southern Hemisphere, H.E.S.S. is best located to observe recently detected DES dSphs compared to other IACTs.

The observations presented here were performed with the full five-telescope array (H.E.S.S. Phase 2) towards a selection of recently discovered dSphs. Dedicated observations were carried out towards Ret II, Tuc II, Tuc III in 2017 and 2018, and Gru II in 2018. Previous observations targeted at another source covered the position of Gru II providing a useful exposure at its nominal position. Given the H.E.S.S. FoV, observations taken towards Tuc III also include Tuc IV. The dedicated observations were taken in the wobble mode where the telescope pointing direction is offset from the nominal target position by an angle between 0.5 and $0.7^{\circ}$. The observations selected for the data analysis meet the standard run selection criteria [30]. Table II 
TABLE II. Table of Milky Way satellites discovered by DES and consistent with dwarf galaxies observed by H.E.S.S. The second to fifth columns give the distance and the galactic coordinates of each object, respectively. The last two columns provide the live time on target acquired by H.E.S.S. in the 2017 and 2018 observation campaigns and the mean zenith angle of observations. Uncertainties on the distance of the systems are of about $10 \%$.

\begin{tabular}{lcccccc}
\hline \hline Source name & RA [hours] & $\begin{array}{c}\text { Dec } \\
\text { [degrees] }\end{array}$ & $\begin{array}{c}\text { Longitude } \\
\text { [degrees] }\end{array}$ & $\begin{array}{c}\text { Latitude } \\
\text { [degrees] }\end{array}$ & $\begin{array}{c}\text { Live time } \\
\text { [hours] }\end{array}$ & $\begin{array}{c}\text { Mean zenith } \\
\text { angle [degrees] }\end{array}$ \\
\hline Reticulum II & $03: 35: 40.8$ & $-54: 03: 00$ & 266.30 & -49.74 & 18.3 & 42.4 \\
Tucana II & $22: 52: 14.4$ & $-58: 34: 12$ & 328.04 & -52.35 & 16.4 & 36.1 \\
Tucana III & $23: 56: 36.0$ & $-59: 36: 00$ & 315.38 & -56.18 & 23.6 & 39.0 \\
Tucana IV & $00: 02: 55.2$ & $-60: 51: 00$ & 313.29 & -55.29 & 12.4 & 39.2 \\
Grus II & $22: 04: 4.8$ & $-46: 26: 24$ & 351.14 & -51.94 & 11.3 & 29.0 \\
\hline \hline
\end{tabular}

summarizes the main observational characteristics of the selected dSphs.

After the calibration of raw shower images, the reconstruction of the direction and energy of gamma rays is performed using a template-fitting technique [31] in which the recorded images are compared to precalculated showers computed from a semianalytical model. This technique achieves an energy resolution of $10 \%$ and an angular resolution of $0.06^{\circ}$ at $68 \%$ containment radius for gamma-ray energies above $200 \mathrm{GeV}$. The results presented here have been cross-checked with a different calibration and analysis chain yielding compatible results [32].

A combined analysis is used to account for the hybrid nature of the observations. Given the configuration of H.E.S.S. Phase II array, a gamma ray can trigger CT5 alone (monoscopic event), or any combination of two of the five telescopes (stereo event). The event reconstruction can be performed in different modes according to the event class. In order to fully benefit from the flexibility of the H.E.S.S. Phase II array, a combined mode exploits both the monoscopic and stereoscopic reconstructions [33]. The best reconstruction among the mono (CT5 only), stereo (CT1-5), or H.E.S.S. Phase I-like (CT1-4) reconstruction of each gamma-ray-like event is selected from a $\chi^{2}$ test. Figure 1 shows each selected dSph the gamma-ray excess sky map for which the residual background computation is determined using the RingBackground technique [30]. Table III summarizes the available gamma-ray statistics in the source and background regions, the relative size of the background region to the signal region, as well as the significance in the source region. No significant gamma-ray excess is found at the position of the $\mathrm{dSph}$ or anywhere in the FoV as shown in Fig. 1.

\section{DATA ANALYSIS}

\section{A. Region of interest and background measurement}

For each selected system, the ON source signal is computed by integrating all the gamma-ray-like events in a disk of angular radius given in the second column of Table III. For Ret II and Tuc II, a ON region of $0.2^{\circ}$ radius is used, divided into two concentric subregions of interest
(ROIs) of $0.1^{\circ}$ width each. The size of the $\mathrm{ON}$ source region is chosen according to the expected DM signal spatial profile versus background as a function of the distance from the object nominal position in order to maximize the sensitivity. In absence of spectroscopic measurements, Tuc III, Tuc IV, and Gru II are considered as pointlike sources for H.E.S.S. and therefore only one ROI with a $0.125^{\circ}$ radius is considered.

The residual background is measured in the OFF-source regions defined according to the multiple-OFF method [34]: for each telescope pointing position of the observations, several regions with same solid angle and shape as for the ROIs are defined at the same distance from the pointing position as the $\mathrm{ON}$ region. By construction, the centers of the OFF regions lie on a circle of radius equal to the distance between the target position and the pointing position, which leads to identical acceptance in the ON and OFF regions. This method yields smaller systematic errors in the analysis than background determination techniques based on OFF measurements in separated dedicated observations of empty fields of view. A disk around the center of the $\mathrm{ON}$ region is excluded for the $\mathrm{OFF}$ measurements, with a radius equal to twice the size of the $\mathrm{ON}$ region radius, so that the expected $\mathrm{DM}$ signal in the OFF regions is negligible. No additional excluded region is used since no VHE gamma-ray sources are detected in the considered FoV. Given the expected source extension of Tuc III and Tuc IV no contamination is expected from one source in the considered signal region of the other. The total number of background events is the sum of all the events that fall in the OFF regions. The parameter $\alpha$ is defined as the ratio between the solid angle size of the OFF and ON regions by $\alpha=\Delta \Omega_{\mathrm{OFF}} / \Delta \Omega_{\mathrm{ON}}$. The excess sky map is obtained by subtracting the total OFF event count weighted by $1 / \alpha$ from the ON event count, and the significance is computed following the statistical approach of Ref. [35]. Figure 1 shows the excess significance sky map for all selected systems. No significant gamma-ray excess is found in the ON source region as well as anywhere else in the sky map. Table III provides the size of the ON source region, the number of ON and OFF events, the $\alpha$ values as well as the excess significance for the full ROIs. 

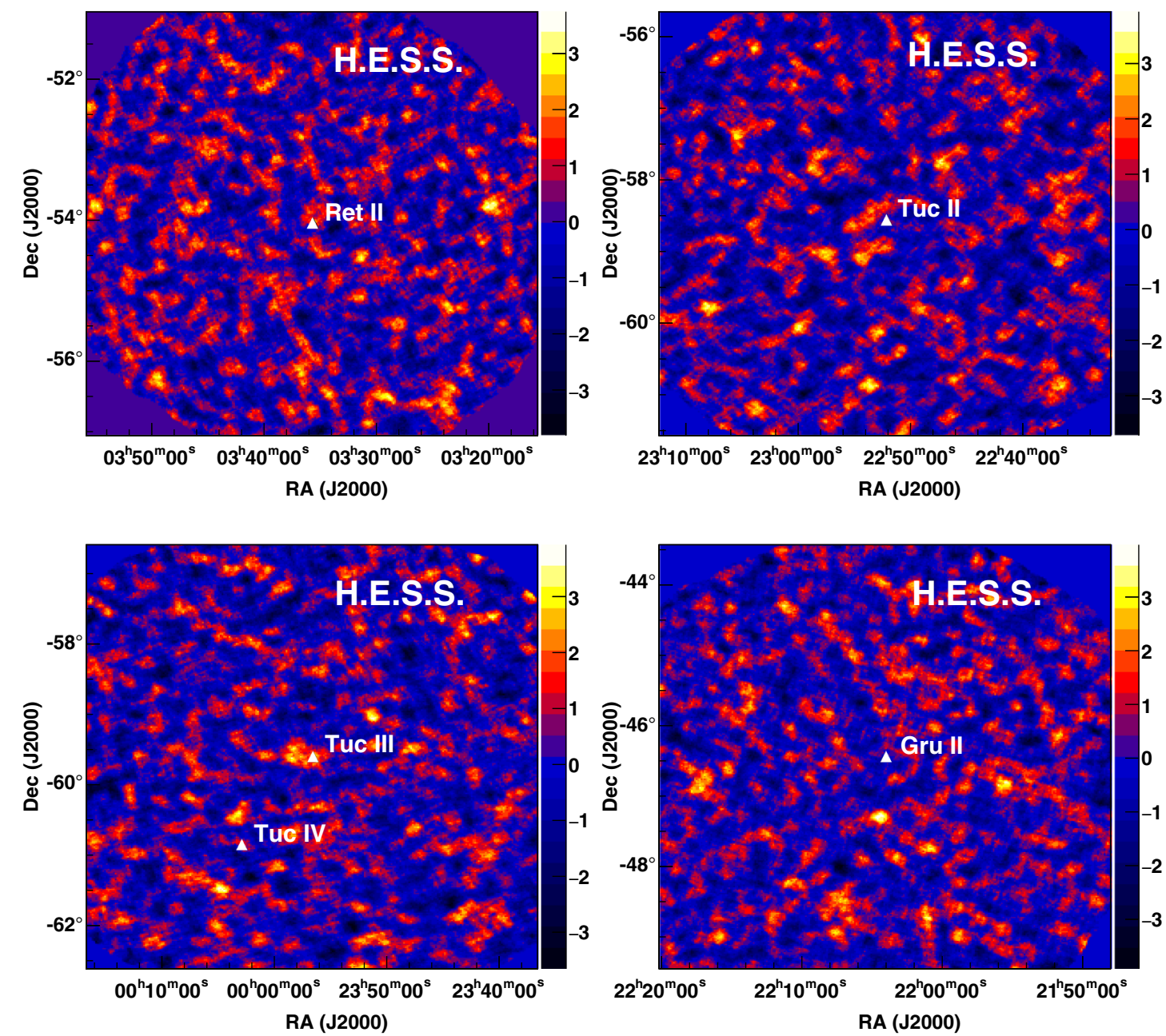

FIG. 1. Excess significance maps in the FoV of Ret II, Tuc II, Tuc III, and Gru II, respectively, in Galactic coordinates. Tuc IV is observed in the FoV of Tuc III. The nominal position of the systems is marked with a white-filled triangle. The color scale gives the significance of the excess in numbers of standard deviations. No significant excess is observed in any of the FoV.

\section{B. Statistical analysis and upper limit computation}

A two-dimensional (2D)-binned Poisson maximum likelihood analysis is used in order to explore the spatial and spectral characteristics of the expected DM signal with respect to the background. The energy range is divided into
68 logarithmically spaced energy bins $i$ between $150 \mathrm{GeV}$ and $63 \mathrm{TeV}$, and the spatial bin $j$ corresponds to the number of ROIs defined for each target. For a given DM mass and annihilation channel, the Poisson likelihood function in the bin $(i, j)$ can be written as

TABLE III. Data analysis results for each selected target. The second column gives the size of the ROI. Count numbers measured in the ON and OFF regions are provided in the third and fourth column, respectively. The fifth and sixth column give the ratio of the solid angle size between the OFF and ON regions averaged over all observations, and the measured excess significance between the ON and OFF counts. For the systems marked with the symbol *, the ROI size is chosen as for pointlike emission searches.

\begin{tabular}{lccccc}
\hline \hline Source name & ON region size [degrees] & $N_{\text {ON }}[$ counts] & $N_{\text {OFF }}[$ counts] & $\bar{\alpha}$ & Significance $[\sigma]$ \\
\hline Reticulum II & 0.200 & 949 & 7926 & 8.0 & -0.9 \\
Tucana II & 0.200 & 1170 & 9704 & 8.0 & -1.0 \\
Tucana III $*$ & 0.125 & 689 & 9816 & 15.0 & 0.9 \\
Tucana IV $*$ & 0.125 & 285 & 6550 & 24.1 & 0.6 \\
Grus II $*$ & 0.125 & 263 & 4491 & 16.0 & -0.8 \\
\hline \hline
\end{tabular}




$$
\begin{aligned}
\mathcal{L}_{\mathrm{ij}}\left(N^{\mathrm{S}}, N^{\mathrm{B}} \mid N_{\mathrm{ON}}, N_{\mathrm{OFF}}, \alpha\right) & \\
= & \frac{\left(N_{\mathrm{ij}}^{\mathrm{S}}+N_{\mathrm{ij}}^{\mathrm{B}}\right)^{N_{\mathrm{ON}, \mathrm{ij}}}}{N_{\mathrm{ON}, \mathrm{ij}} !} e^{-\left(N_{\mathrm{ij}}^{\mathrm{S}}+N_{\mathrm{ij}}^{\mathrm{B}}\right)} \\
& \times \frac{\left(N_{\mathrm{ij}}^{\mathrm{S}^{\prime}}+\alpha_{\mathrm{j}} N_{\mathrm{ij}}^{\mathrm{B}}\right)^{N_{\mathrm{OFF}, \mathrm{ij}}}}{N_{\mathrm{OFF}, \mathrm{ij}} !} e^{-\left(N_{\mathrm{ij}}^{\mathrm{S}^{\prime}}+\alpha_{\mathrm{j}} N_{\mathrm{ij}}^{\mathrm{B}}\right)} .
\end{aligned}
$$

$N_{\mathrm{ON}, \mathrm{ij}}$ and $N_{\mathrm{OFF}, \mathrm{ij}}$ stand for the number of measured events in the ON and OFF regions, respectively. $N_{\mathrm{ij}}^{\mathrm{B}}$ is the expected number of background events in the ON region. $\alpha_{\mathrm{j}}$ is defined as the ratio of the solid angle of the OFF and ON regions for the bin $j . N_{\mathrm{ij}}^{\mathrm{S}}$ and $N_{\mathrm{ij}}^{\mathrm{S}^{\prime}}$ correspond to the number of DM signal events expected in the ON and OFF regions, respectively. They are computed by folding the theoretical DM flux with the energy-dependent acceptance and energy resolution of H.E.S.S. for the considered data set. The continuum signal spectra are extracted from Ref. [36], while the monoenergetic gamma-line signal is a Dirac delta function. The energy resolution of H.E.S.S. is represented by a Gaussian function with a width of $\sigma_{\mathrm{E}} / E=10 \%$. The dependence of the energy resolution on the observational parameters (mean zenith angle, optical efficiency, off-axis angle) and the analysis selection cuts have a negligible impact on the results. The same likelihood analysis technique is applied to look both for the continuum and gamma-line signals.

As discussed in Sec. IVA, $N_{\mathrm{ij}}^{\mathrm{S}^{\prime}}$ can be safely taken to $N_{\mathrm{ij}}^{\mathrm{S}^{\prime}} \equiv 0$. The total likelihood $\mathcal{L}$ is defined as the product of the $\mathcal{L}_{\mathrm{ij}}$ over the $i j$ bins. Since no significant excess between the ON and OFF regions is found in any of the considered systems, upper limits can be computed for any DM mass from a likelihood ratio test statistic (TS) [37] given by:

$$
T S=\left\{\begin{array}{ll}
-2 \ln \frac{\mathcal{L}\left(N^{S}(\langle\sigma v\rangle)\right) \widehat{\left.\widehat{N^{B}}(\langle\sigma v\rangle)\right)}}{\widehat{\widehat{L}\left(0, N^{B}(0)\right)}} & N^{S}(\widehat{\langle\sigma v\rangle})<0 \\
-2 \ln \frac{\left.\mathcal{L}\left(N^{S}(\langle\sigma v\rangle), \widehat{N^{B}}(\langle\sigma v\rangle)\right)\right)}{\mathcal{L}\left(N^{S}(\langle\sigma v\rangle), N^{B}\right)} & 0 \leq N^{S}(\widehat{\langle\sigma v\rangle}) \leq N^{S}(\langle\sigma v\rangle) \\
0 & N^{S}(\widehat{\langle\sigma v\rangle})>N^{S}(\langle\sigma v\rangle)
\end{array} .\right.
$$

$\widehat{\widehat{N_{\mathrm{ij}}^{\mathrm{B}}}}$ is obtained through a conditional maximization by solving $d \mathcal{L} / d N_{\mathrm{ij}}^{\mathrm{B}}=0$, while $N_{\mathrm{ij}}^{\mathrm{S}}(\widehat{\langle\sigma v\rangle})$ and $\widehat{N_{\mathrm{ij}}^{\mathrm{B}}}$ are computed using an unconditional numerical maximization. The procedure described in Ref. [37] has been followed in order to compute upper limits for positive signals. The value of the velocity-weighted annihilation cross section $\langle\sigma v\rangle$ for which $\Delta \mathrm{TS}=2.71$ from the minimum provides one-sided 95\% confidence level (C.L.) upper limits on $\langle\sigma v\rangle$.
Due to the finite number of stellar tracers of the DMinduced gravitational potential in a given DES system $k$, the $J$-factor can be treated as a statistical variable. Its uncertainty is modeled as a nuisance parameter which follows a log-normal distribution:

$$
\begin{aligned}
\mathcal{L}_{\mathrm{k}}^{\mathrm{J}}\left(J \mid \bar{J}, \sigma_{J}\right)= & \frac{1}{\sqrt{2 \pi} \sigma_{J} \log (10) \times J} \\
& \times \exp \left(-\frac{\left(\log _{10} J-\overline{\log _{10} J}\right)^{2}}{2 \sigma_{J}^{2}}\right),
\end{aligned}
$$

with mean $\bar{J}$ and width $\sigma_{\mathrm{J}}$ taken from literature (see Table I). The $J$-factor, $\hat{J}$, that maximizes Eq. (4) is derived and then included in the likelihood test as $N_{\mathrm{S}} \rightarrow N_{\mathrm{S}} \hat{J} / \bar{J}$.

\section{RESULTS}

\section{A. Upper limits on individual systems}

Since no significant excess is found in the selected DES dSphs in any ROI, upper limits at 95\% C.L. on $\langle\sigma v\rangle$ versus the DM mass are derived for each target following Eq. (3). The upper limits as a function of the DM mass are shown in Fig. 2 for the $W^{+} W^{-}$annihilation channel. The observed limits are plotted together with the mean expected limits and the $1 \sigma$ and $2 \sigma$ containment bands. Mean expected limits and statistical uncertainty bands are obtained from 100 Poisson realizations of the background in the $\mathrm{ON}$ and OFF regions, respectively. The mean expected limits are given by the mean of the distribution of $\log _{10}(\langle\sigma v\rangle)$ obtained in the realizations and the containment bands by its standard deviation.

The best observed limits are obtained for Ret II. They reach $\langle\sigma v\rangle \simeq 1 \times 10^{-23} \mathrm{~cm}^{3} \mathrm{~s}^{-1}$ for a $1.5 \mathrm{TeV}$ DM mass in the $W^{+} W^{-}$annihilation channel. In the case of Tuc III, they reach $\langle\sigma v\rangle \simeq 3 \times 10^{-23} \mathrm{~cm}^{3} \mathrm{~s}^{-1}$ for a $1.5 \mathrm{TeV} \mathrm{DM}$ mass. Assuming the lower value of the $J$-factor for Tuc III, the limits degrade by a factor of about 40 . The limits on Tuc IV and Gru II are less constraining due to smaller $J$-factors and datasets.

In addition, a search for monoenergetic gamma-ray lines has been performed on the five targets. The 95\% C.L. observed and mean expected limits together with the containment bands are shown in Fig. 3 for the five targets. For Ret II, the observed limit reaches $\langle\sigma v\rangle \simeq 8 \times 10^{-26} \mathrm{~cm}^{3} \mathrm{~s}^{-1}$ for a $1.5 \mathrm{TeV}$ DM mass.

The search for a DM signal has also been performed in the annihilation channels $Z Z, b \bar{b}, t \bar{t}, e^{+} e^{-}, \mu^{+} \mu^{-}$, and $\tau^{+} \tau^{-}$. The $95 \%$ C.L. upper limits on $\langle\sigma v\rangle$ as a function of $m_{\mathrm{DM}}$ are shown in Fig. 4 for the most promising target, Ret II.

The impact of the uncertainty on the $J$-factor is computed for Ret II and Tuc II, as shown in Fig. 5 for the $W^{+} W^{-}$channel. The limits degrade by a factor of about six and 12 for Ret II and Tuc II, respectively. 

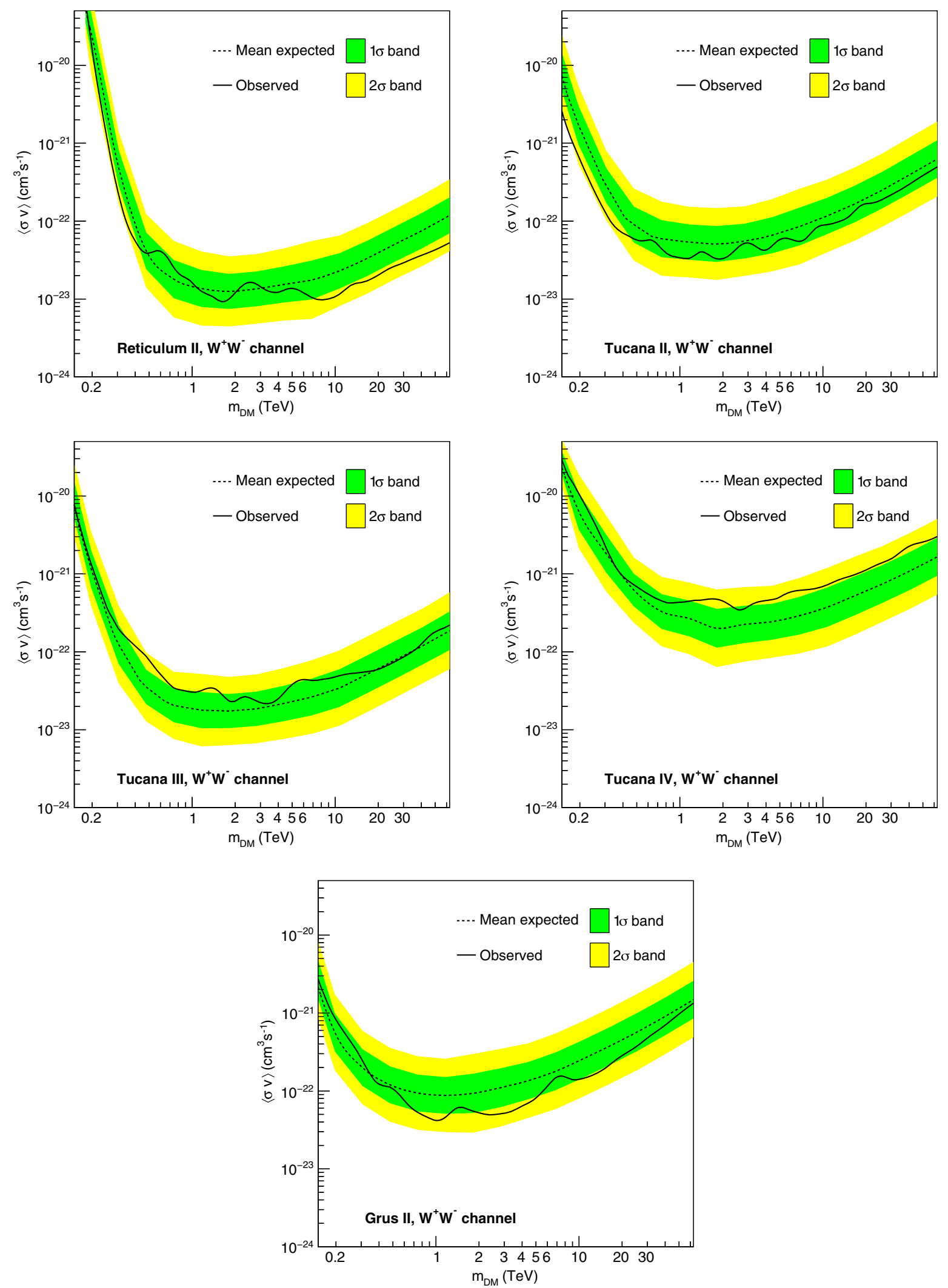

FIG. 2. 95\% C.L. upper limits on the annihilation cross section $\langle\sigma v\rangle$ for Ret II, Tuc II, Tuc III, Tuc IV, and Gru II, in the $W^{+} W^{-}$ annihilation channel without the uncertainty on the $J$-factor. Observed limits (solid lines) together with mean expected limits (dashed line) and the $1 \sigma$ (green area) and $2 \sigma$ (yellow area) containment bands are shown. 

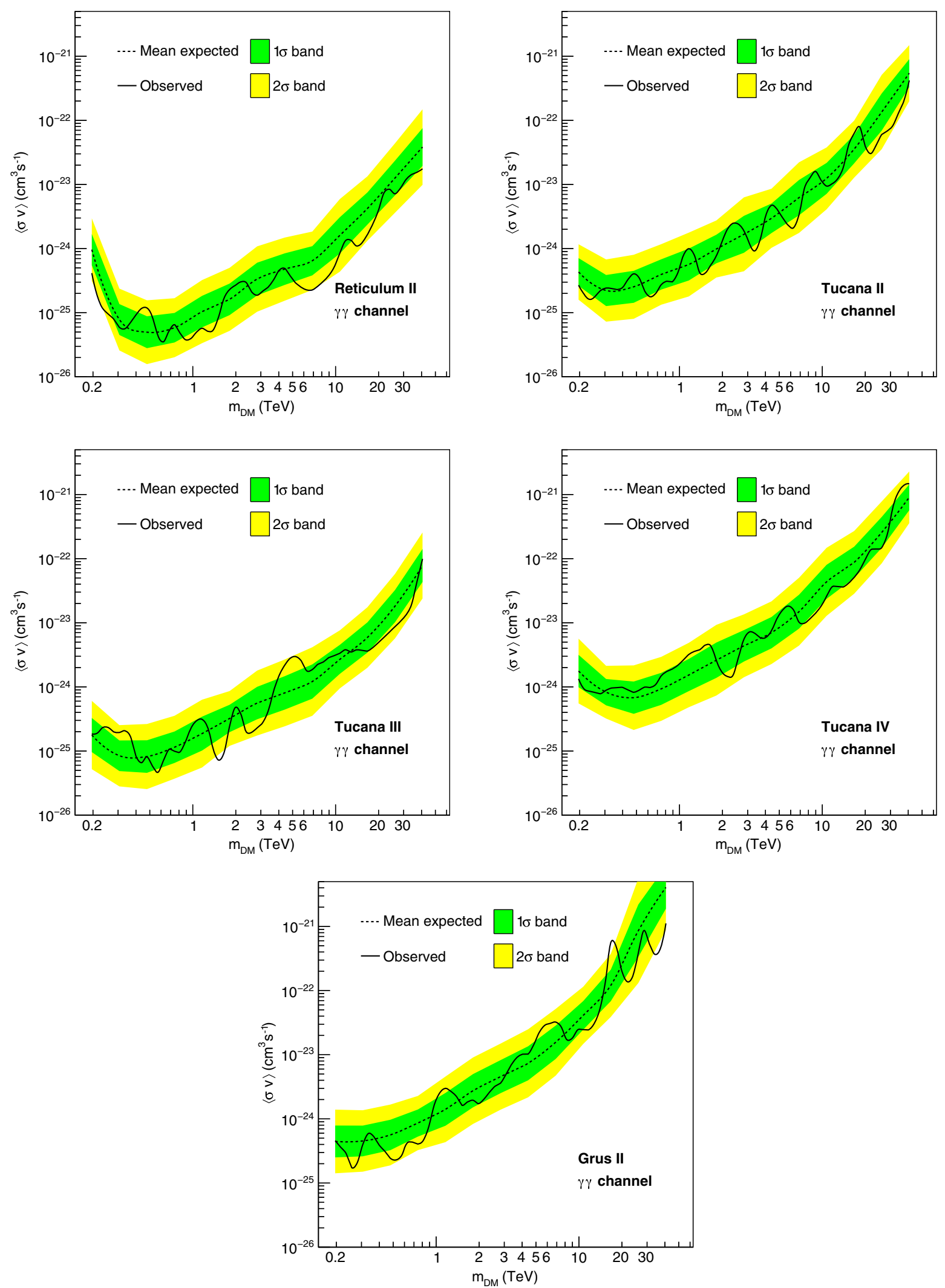

FIG. 3. 95\% C.L. upper limits on the annihilation cross section $\langle\sigma v\rangle$ versus the DM mass $m_{\mathrm{DM}}$ for Ret II, Tuc II, Tuc III, Tuc IV, Gru II, in the $\gamma \gamma$ annihilation channel, without the uncertainty on the $J$-factor. Observed limits (solid lines) together with mean expected limits (dashed lines), and the $1 \sigma$ (green area) and $2 \sigma$ (yellow area) containment bands are shown. 

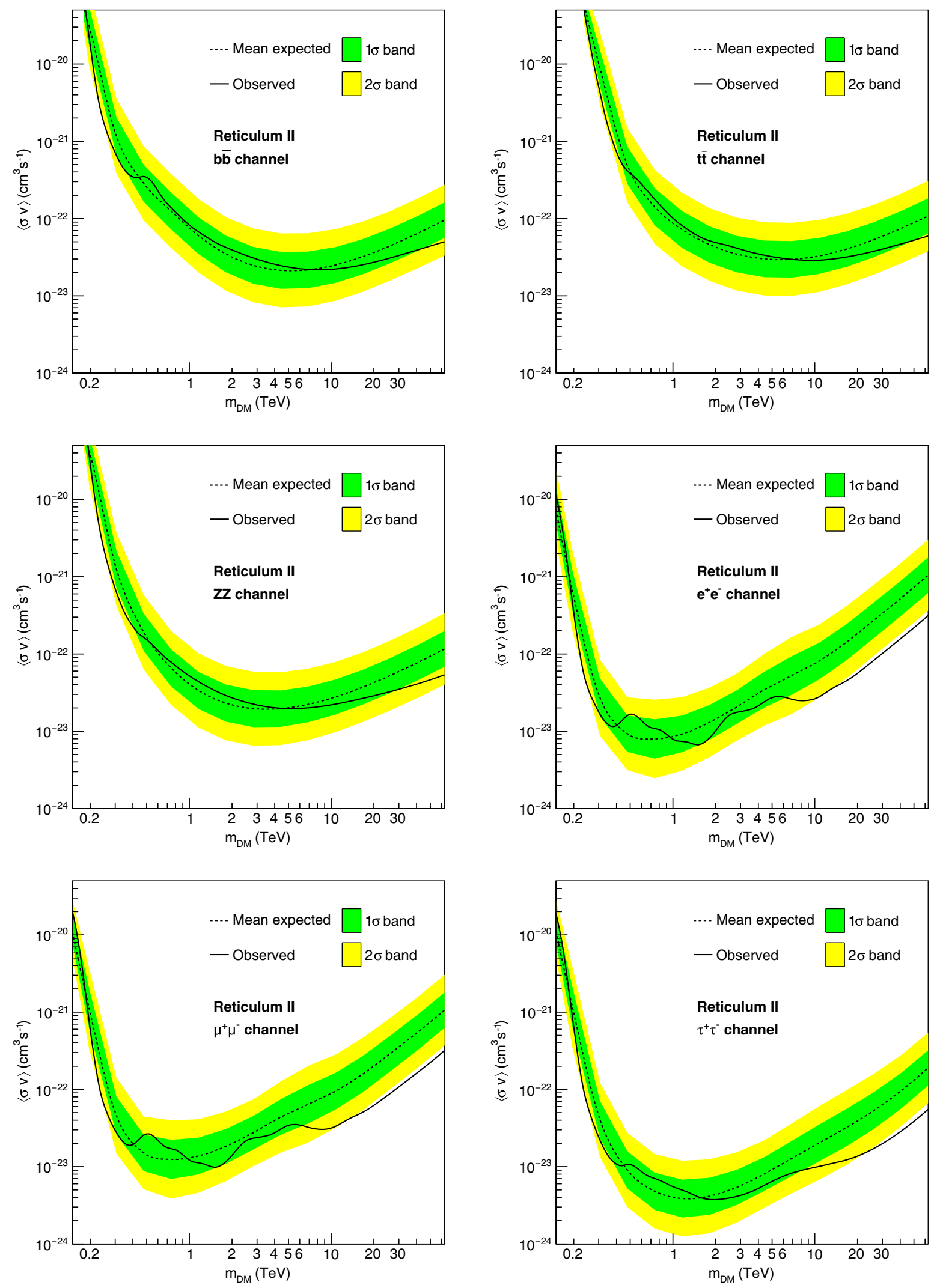

FIG. 4. 95\% C.L. upper limits on the annihilation cross section $\langle\sigma v\rangle$ for Ret II in the $b \bar{b}, t \bar{t}, Z Z, e^{+} e^{-}, \mu^{+} \mu^{-}, \tau^{+} \tau^{-}$annihilation channels, respectively, without the uncertainty on the $J$-factor. Observed limits (solid lines) together with mean expected limits (dashed lines) and the $1 \sigma$ (green area) and $2 \sigma$ (yellow area) containment bands are shown. The limits for the other targets can be found in Figs. 9-12 in the Appendix. 

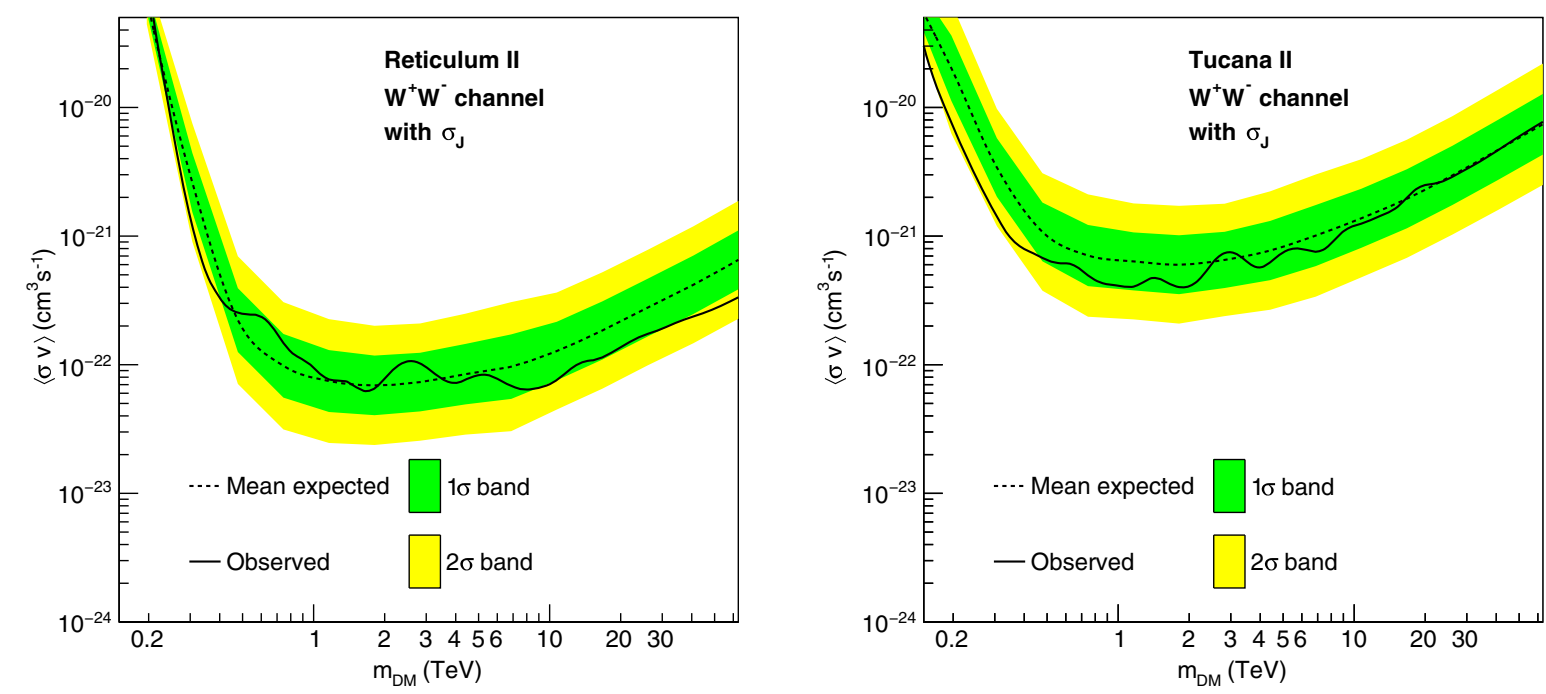

FIG. 5. 95\% C.L. upper limits on the annihilation cross section $\langle\sigma v\rangle$ for Ret II (left panel) and Tuc II (right panel) in the $W^{+} W^{-}$ annihilation channel including the uncertainties on the $J$-factor. Observed limits (solid lines) together with mean expected limits (dashed lines) with $1 \sigma$ (green area) and $2 \sigma$ (yellow area) containment bands are shown.
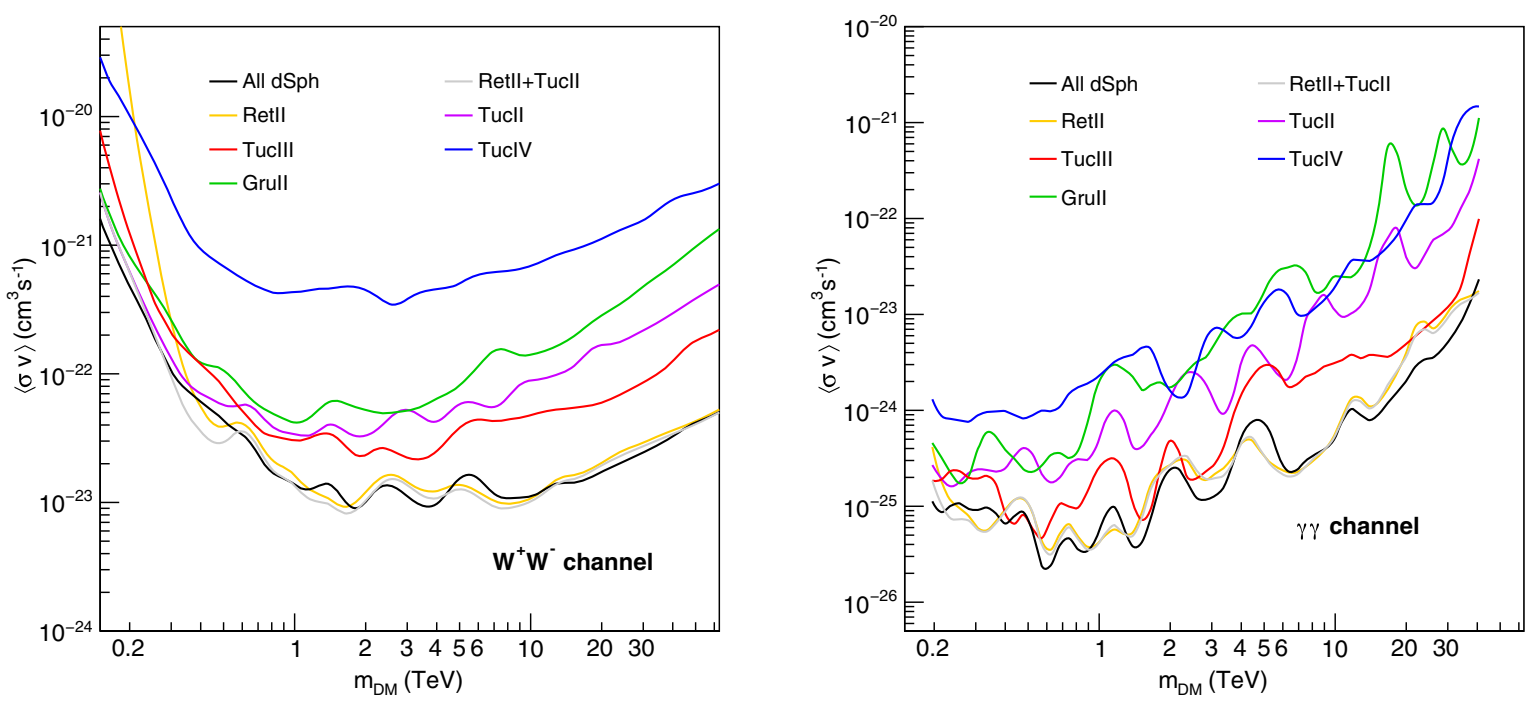

FIG. 6. 95\% C.L. observed upper limits on the annihilation cross section $\langle\sigma v\rangle$ versus the DM mass $m_{\mathrm{DM}}$ for the combined analysis in the $W^{+} W^{-}$(left panel) and $\gamma \gamma$ (right panel) annihilation channels, respectively, without the uncertainty on the $J$-factor.

\section{B. Combined upper limits}

The hypothesis that all targets are in fact gamma-ray emitters, but too faint to be seen with the given exposure, was tested and no overall significant excess was found. The combination was performed at the likelihood level, where the total likelihood function writes

$$
\mathcal{L}_{\text {joint }}=\prod_{\mathrm{k}=1}^{N_{\text {targets }}} \mathcal{L}_{\mathrm{k}},
$$

where $\mathcal{L}_{\mathrm{k}}$ is the likelihood of each target $k$. A strict jointlikelihood maximization was not performed, but the likelihoods were maximized beforehand. The combined observed limits at 95\% C.L. on the $W^{+} W^{-}$and $\gamma \gamma$ channels are shown in the left and right panels of Fig. 6, respectively. For a $1.5 \mathrm{TeV}$ DM mass, they reach $\langle\sigma v\rangle \simeq 1 \times 10^{-23} \mathrm{~cm}^{3} \mathrm{~s}^{-1}$ and $4 \times 10^{-26} \mathrm{~cm}^{3} \mathrm{~s}^{-1}$ in the $W^{+} W^{-}$and $\gamma \gamma$ annihilation channels, respectively. These results degrade of about a factor seven when the uncertainty on the $J$-factor is included.

The combination of the two confirmed dwarf galaxies, Ret II and Tuc II, is shown as well as the combination of all the five objects. In the former case the limits are driven by Ret II limits, while in the latter the impact of Tuc III is also significant. The combined 95\% C.L. observed limits of the 


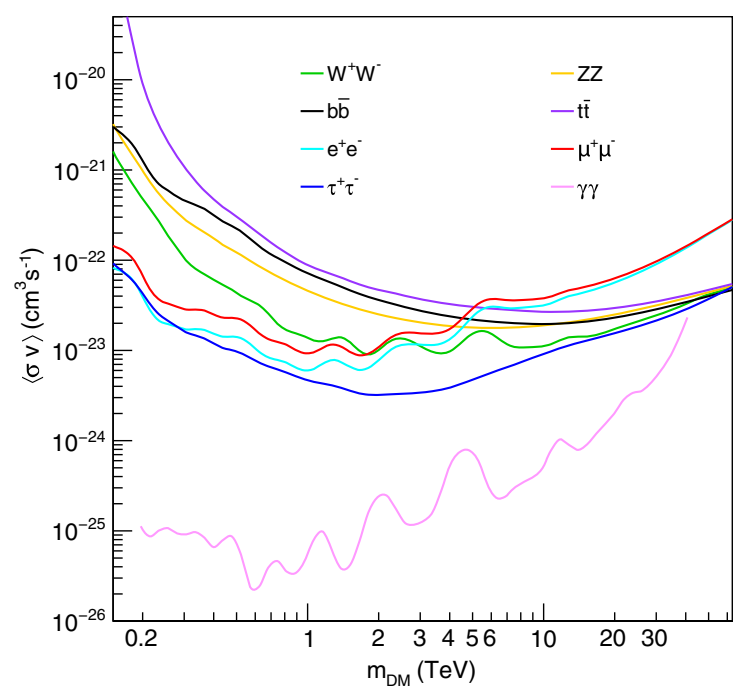

FIG. 7. Combined 95\% C.L. observed upper limits on the annihilation cross section $\langle\sigma v\rangle$ versus the DM mass $m_{\mathrm{DM}}$ for the combined analysis in the $b \bar{b}, t \bar{t}, W^{+} W^{-}, Z Z, e^{+} e^{-}, \mu^{+} \mu^{-}, \tau^{+} \tau^{-}$, and $\gamma \gamma$ annihilation channels, respectively, without the uncertainty on the $J$-factor.

five objects are plotted together in Fig. 7 for various annihilation channels.

Constraints on $\langle\sigma v\rangle$ from various experiments are compared in Fig. 8 for the $W^{+} W^{-}$(left) and $\gamma \gamma$ (right) annihilation channels, respectively. The results obtained by H.E.S.S. in this work combining the five selected DES $\mathrm{dSphs}$, with and without including the uncertainty on the $J$-factor are shown together with previous H.E.S.S. results obtained on a selection of classical dSphs [38] including the uncertainty on the $J$-factor. ${ }^{1}$ The results from MAGIC on Segue $1^{2}$ with (dashed) and without (solid) the uncertainty on the $J$-factor are extracted from Ref. [40] and Ref. [41], respectively. Results obtained by VERITAS from a combination of five dSphs including Segue 1 [42], with and without uncertainty on the $J$-factor are plotted together with the stacked limits on $15 \mathrm{dSphs}$ obtained by Fermi-LAT with the uncertainty on the $J$-factor [43]. The results obtained from the HAWC experiment on 15 targets [44] without the uncertainty on the $J$-factor as well as results that do not include Triangulum $\mathrm{II}^{3}$ with and without uncertainty on the

\footnotetext{
${ }^{1}$ These results are quoted with and without Sagittarius dSph given that the determination of its dark matter profile is challenging for this tidally disrupted system. See, for instance, Ref. [4].

${ }^{2}$ The large $J$-factor value used for Segue 1 in the above mentioned results can be overestimated by a factor up to 100 [39].

${ }^{3}$ An accurate determination of the $J$-factor of Triangulum II is difficult due to the reduced number of detected member stars (13) and possible tidal stripping [45]. The total $J$-factor of Triangulum II used in Ref. [44] is $\log _{10}\left(\mathrm{~J} / \mathrm{GeV}^{2} \mathrm{~cm}^{-5}\right)=20.44$. Such a large value is quite speculative and may have been artificially obtained by the presence of a binary star with variable radial velocity [45]. The reduced number of member stars makes also the $J$-factor determination more prone to systematic uncertainties.
}
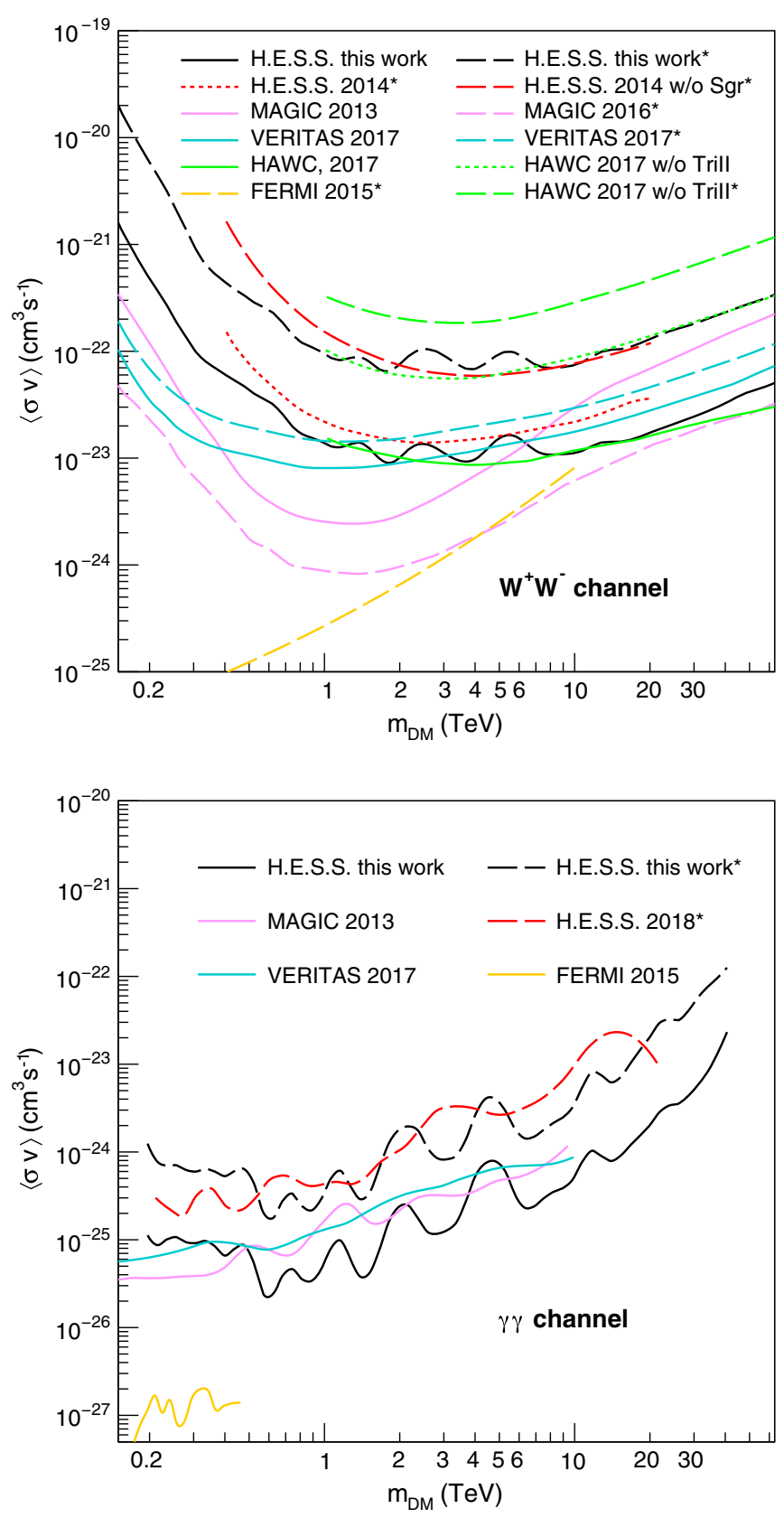

FIG. 8. Comparison of the observed limits versus the DM mass $m_{\mathrm{DM}}$ in the $W^{+} W^{-}$(left panel) and $\gamma \gamma$ (right panel) annihilation channels, respectively. Constraints are shown for HAWC (507 days of data taking, combination of 15 galaxies) with and without Triangulum II [44], for Fermi-LAT (6 years of data taking, combination of 15 galaxies for $W^{+} W^{-}$[43] and 5.8 years of data taken in the Galactic Center region [47]), for H.E.S.S. (140 h, combination of five classical galaxies) with and without 90 hours of observations on Sagittarius dSph $[38,46]$, for MAGIC (160 h on Segue I) $[40,41]$, and for VERITAS (128 h, combination of five galaxies) [42]. The results marked with $*$ include the uncertainty on the $J$-factor. See text for more details. 
$J$-factor are also shown. In the $\gamma \gamma$ annihilation channel, the previous H.E.S.S. results on a selection of classical dSphs are extracted from Ref. [46]. The results on Segue 1 from MAGIC [40] as well as those by VERITAS on five dSphs including Segue 1 [42], without uncertainty on the $J$-factor, are plotted. The Fermi-LAT limits on the Galactic Center [47] are also displayed. The constraints obtained in the $\gamma \gamma$ annihilation channel from H.E.S.S. are particularly relevant to constraint DM models with enhanced linelike signals in the $\mathrm{TeV}$ mass range.

\section{SUMMARY AND DISCUSSIONS}

H.E.S.S. is the first IACT to observe a selection of ultrafaint dwarf satellites of the Milky Way recently discovered by DES to search for a DM annihilation signal with the highest sensitivity among IACTs towards these objects given its position in the Southern hemisphere. The exposure towards the five selected targets, Ret II, Tuc II, Tuc III, Tuc IV, and Gru II, amounts to about 80 hours of live time. In absence of a significant excess in any of the object FoV, 95\% C.L. upper limits have been derived on the DM annihilation cross section as a function of the DM mass in various annihilation channels. The strongest limits from an individual object are obtained for Ret II. Assuming no uncertainty in the $J$-factor, they reach $\langle\sigma v\rangle \simeq$ $1 \times 10^{-23} \mathrm{~cm}^{3} \mathrm{~s}^{-1}$ and $8 \times 10^{-26} \mathrm{~cm}^{3} \mathrm{~s}^{-1}$ in the $W^{+} W^{-}$ and $\gamma \gamma$ annihilation channels, respectively, for a $1.5 \mathrm{TeV}$ DM mass. Assuming an uncertainty on the $J$-factor, the limits degrade by about a factor seven. Using a lower mean value for Ret II $J$-factor would degrade the limits accordingly. The limits from the combined analysis of the five targets are dominated by Ret II limits assuming the conservative $J$-factor value for Tuc II. $\ln$ the $W^{+} W^{-}$ annihilation channel they reach about the same values within the statistical fluctuations. They go slightly down to $\langle\sigma v\rangle \simeq 9 \times 10^{-24} \mathrm{~cm}^{3} \mathrm{~s}^{-1}$ for a $1.5 \mathrm{TeV}$ DM mass when considering only Ret II and Tuc II. The combined limits on the five targets in $\gamma \gamma$ reach $\langle\sigma v\rangle \simeq 4 \times 10^{-26} \mathrm{~cm}^{3} \mathrm{~s}^{-1}$ for a $1.5 \mathrm{TeV} \mathrm{DM}$. Including the $J$-factor uncertainty possibly degrades the combined limits up to a factor of about seven.

The uncertainty on the $J$-factor for ultrafaint systems is challenging to measure or predict. The limits derived in this work and similar studies are strongly dependent on the choice of the $J$-factor mean value and its uncertainty for a given system. Most often only the statistical uncertainty on the $J$-factor coming from the finite number of stellar tracers is considered. Only a few studies investigate the impact of the assumptions made to derive the $J$-factor value (see, for instance, Refs. [28,48]). Among the possible sources of systematic uncertainty in the $J$-factor determination [29] are the assumptions of a single stellar population or spherical symmetry, a constant velocity anisotropy, and the absence of tidal stripping. The selection of member stars for ultrafaint systems is also complex due to the difficulty to distinguish member stars from interlopers in the foreground. Another caveat when comparing limits including the uncertainty on the $J$-factors from one experiment to another is the treatment of the $J$-factor uncertainty in the derivation of the limits. Here a log-normal distribution is taken for the $J$-factor likelihood function while some studies use a modified log-normal distribution [26,41]. In addition, the procedure to derive the maximized $J$-factor may differ from one study to another.

The new results obtained by H.E.S.S. are among the most constraining in the $\gamma \gamma$ annihilation channel above $500 \mathrm{GeV}$. They are comparable to VERITAS and HAWC limits in the $W^{+} W^{-}$annihilation channel in the multi-GeV and multiTeV DM mass ranges, respectively. The constraints obtained in the $\gamma \gamma$ annihilation channel are particularly relevant in the context of DM models with enhanced line signals in the TeV DM mass ranges. Among them are models with gamma-ray boxes [49], scalar [50], and Dirac [51] DM models, as well as the canonical Majorana DM triplet fermion known as the Wino [52,53], and the DM doublet fermion known as the Higgsino [54-56].

The constraints obtained in this work are competitive with other experiments. While the likelihood function definition, the test statistics and the background determination technique may vary from one experiment to another, the results complement each other showing the importance of having instruments with different characteristics that observe a different selection of targets. The IACTs are powerful instruments to investigate the multi-TeV DM not accessible to Fermi-LAT. This is particularly true when it comes to search for TeV DM-induced spectral features close to the DM mass. In the $\gamma \gamma$ channel where the expected signal is very sharp the Fermi-LAT sensitivity range cannot extend beyond a few hundred $\mathrm{GeV}$ where its detected photon statistics are very low. The excellent energy resolution of H.E.S.S. is crucial to search for monoenergetic signals expected in the $\gamma \gamma$ annihilation channel. In addition, the H.E.S.S. instrument performance enable to cover the broadest DM mass range among the IACTs for linelike signal searches.

Future studies would greatly benefit from high-quality stellar kinematic datasets towards the most promising ultrafaint dSph satellites discovered by DES, such as Tuc III, Tuc IV, and Gru II in order to improve the knowledge of the DM density distribution in these objects. 


\section{ACKNOWLEDGMENTS}

The support of the Namibian authorities and of the University of Namibia in facilitating the construction and operation of H.E.S.S. is gratefully acknowledged, as is the support by the German Ministry for Education and Research (BMBF), the Max Planck Society, the German Research Foundation (DFG), the Helmholtz Association, the Alexander von Humboldt Foundation, the French Ministry of Higher Education, Research and Innovation, the Centre National de la Recherche Scientifique (CNRS/ IN2P3 and CNRS/INSU), the Commissariat à l'énergie atomique et aux énergies alternatives (CEA), the U.K. Science and Technology Facilities Council (STFC), the Knut and Alice Wallenberg Foundation, the National Science Centre, Poland Grant No. 2016/22/M/ST9/
00382, the South African Department of Science and Technology and National Research Foundation, the University of Namibia, the National Commission on Research, Science \& Technology of Namibia (NCRST), the Austrian Federal Ministry of Education, Science and Research and the Austrian Science Fund (FWF), the Australian Research Council (ARC), the Japan Society for the Promotion of Science and by the University of Amsterdam. We appreciate the excellent work of the technical support staff in Berlin, Zeuthen, Heidelberg, Palaiseau, Paris, Saclay, Tubingen and in Namibia in the construction and operation of the equipment. This work benefited from services provided by the H.E.S.S. Virtual Organisation, supported by the national resource providers of the EGI Federation. 


\section{APPENDIX: CONSTRAINTS FOR ADDITIONAL ANNIHILATION CHANNELS TOWARDS TUC II, TUC III, TUC IV AND GRU II}
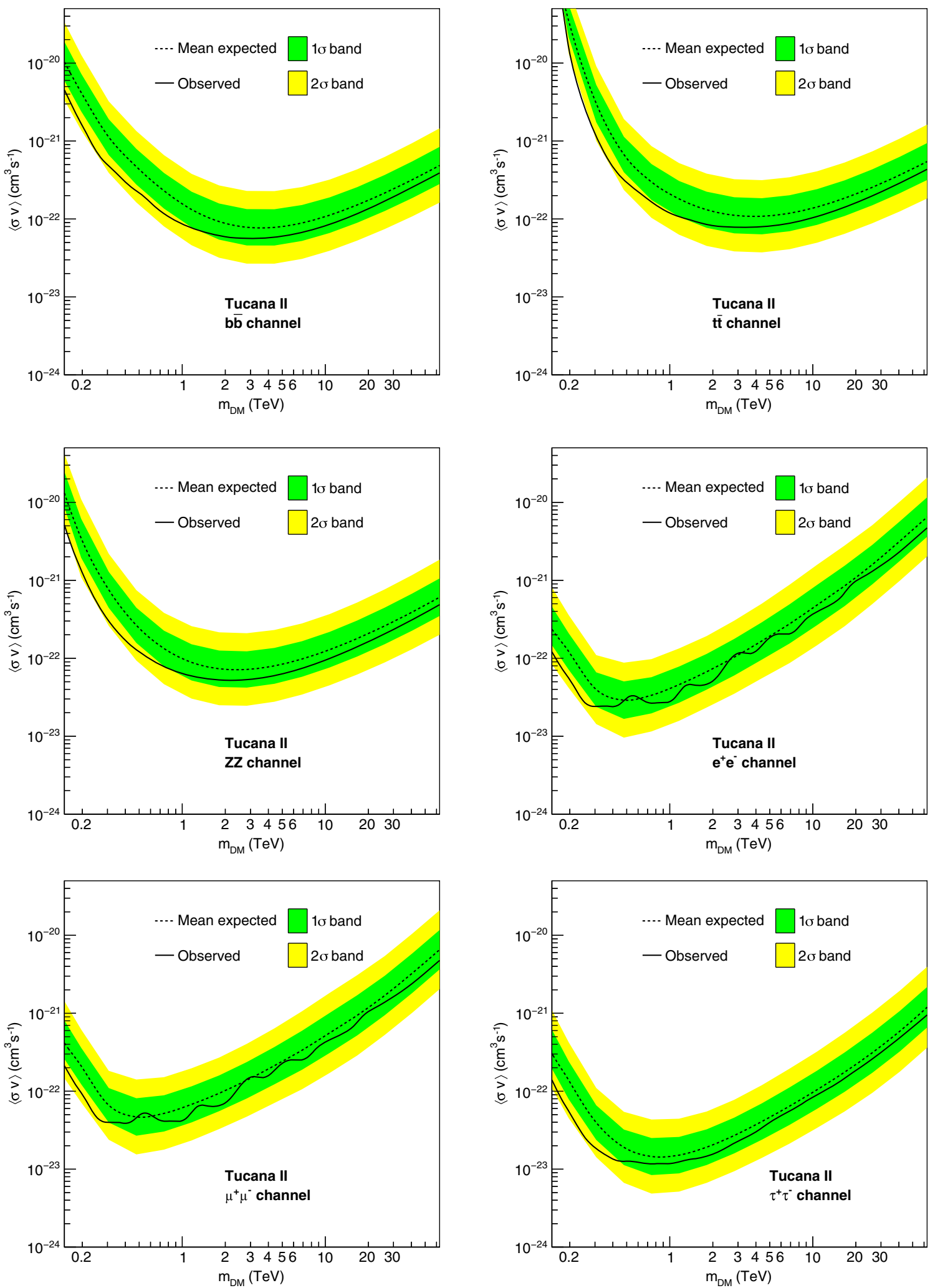

FIG. 9. $95 \%$ C.L. upper limits on the annihilation cross section $\langle\sigma v\rangle$ for Tuc II in the $b \bar{b}, t \bar{t}, Z Z, e^{+} e^{-}, \mu^{+} \mu^{-}, \tau^{+} \tau^{-}$annihilation channels, respectively, without the uncertainty on the $J$-factor. Observed limits (solid lines) together with mean expected limits (dashed line) and the $1 \sigma$ (green area) and $2 \sigma$ (yellow area) containment bands are shown, respectively. 

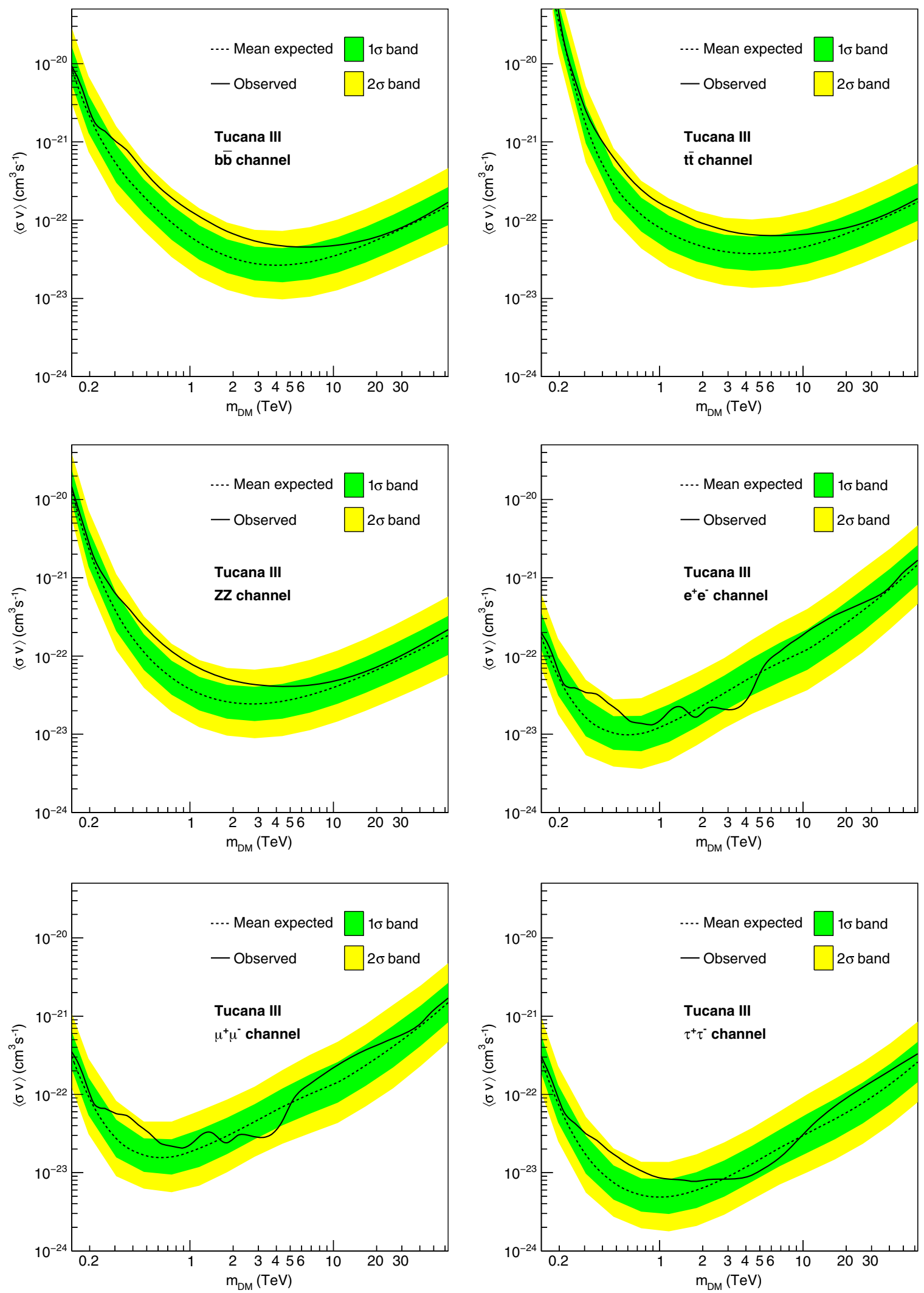

FIG. 10. 95\% C.L. upper limits on the annihilation cross section $\langle\sigma v\rangle$ for Tuc III in the $b \bar{b}, t \bar{t}, Z Z, e^{+} e^{-}, \mu^{+} \mu^{-}, \tau^{+} \tau^{-}$annihilation channels, respectively, without the uncertainty on the $J$-factor. Observed limits (solid lines) together with mean expected limits (dashed line) and the $1 \sigma$ (green area) and $2 \sigma$ (yellow area) containment bands are shown, respectively. 

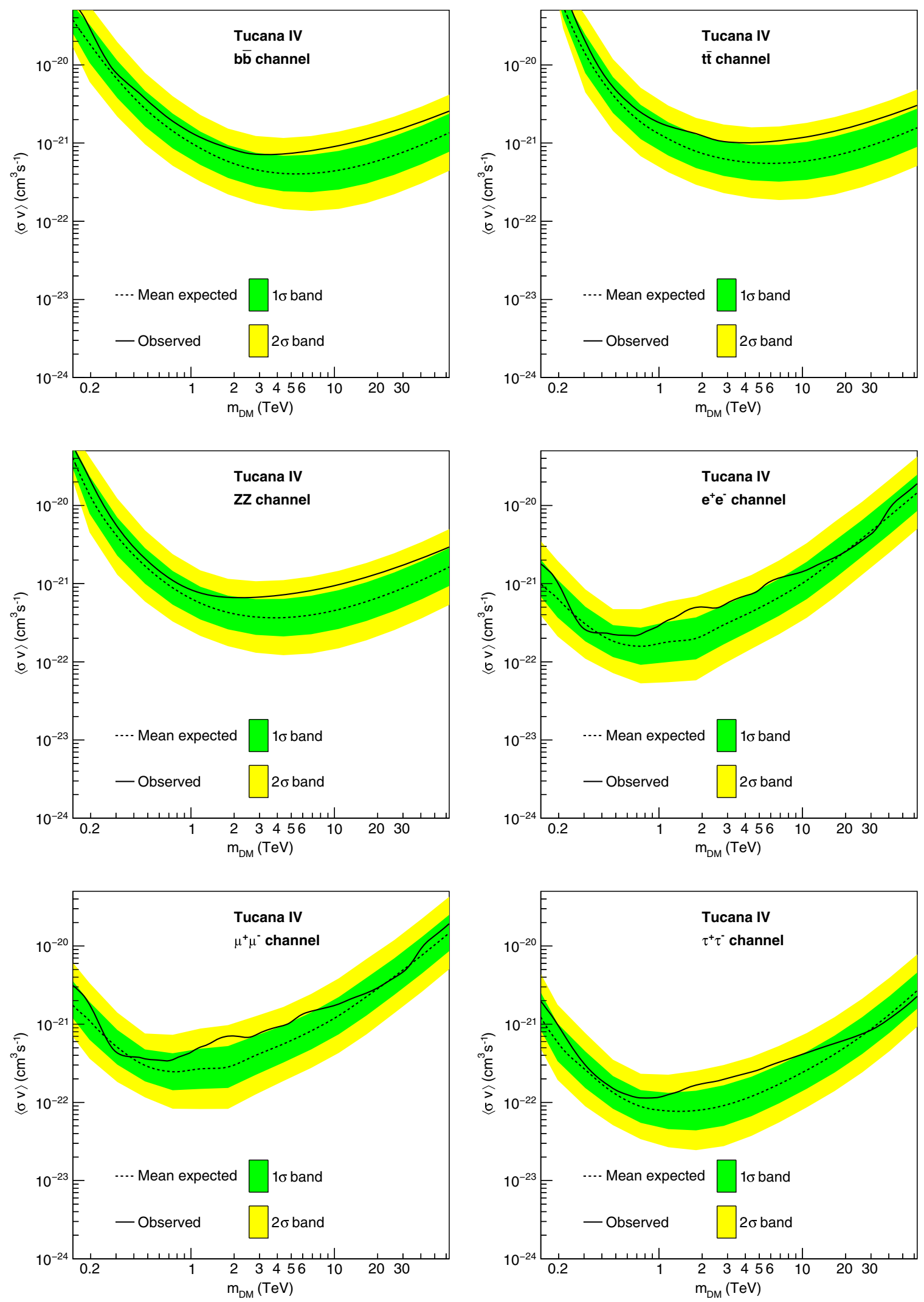

FIG. 11. 95\% C.L. upper limits on the annihilation cross section $\langle\sigma v\rangle$ for Tuc IV in the $b \bar{b}, t \bar{t}, Z Z, e^{+} e^{-}, \mu^{+} \mu^{-}, \tau^{+} \tau^{-}$annihilation channels, respectively, without the uncertainty on the $J$-factor. Observed limits (solid lines) together with mean expected limits (dashed line) and the $1 \sigma$ (green area) and $2 \sigma$ (yellow area) containment bands are shown, respectively. 

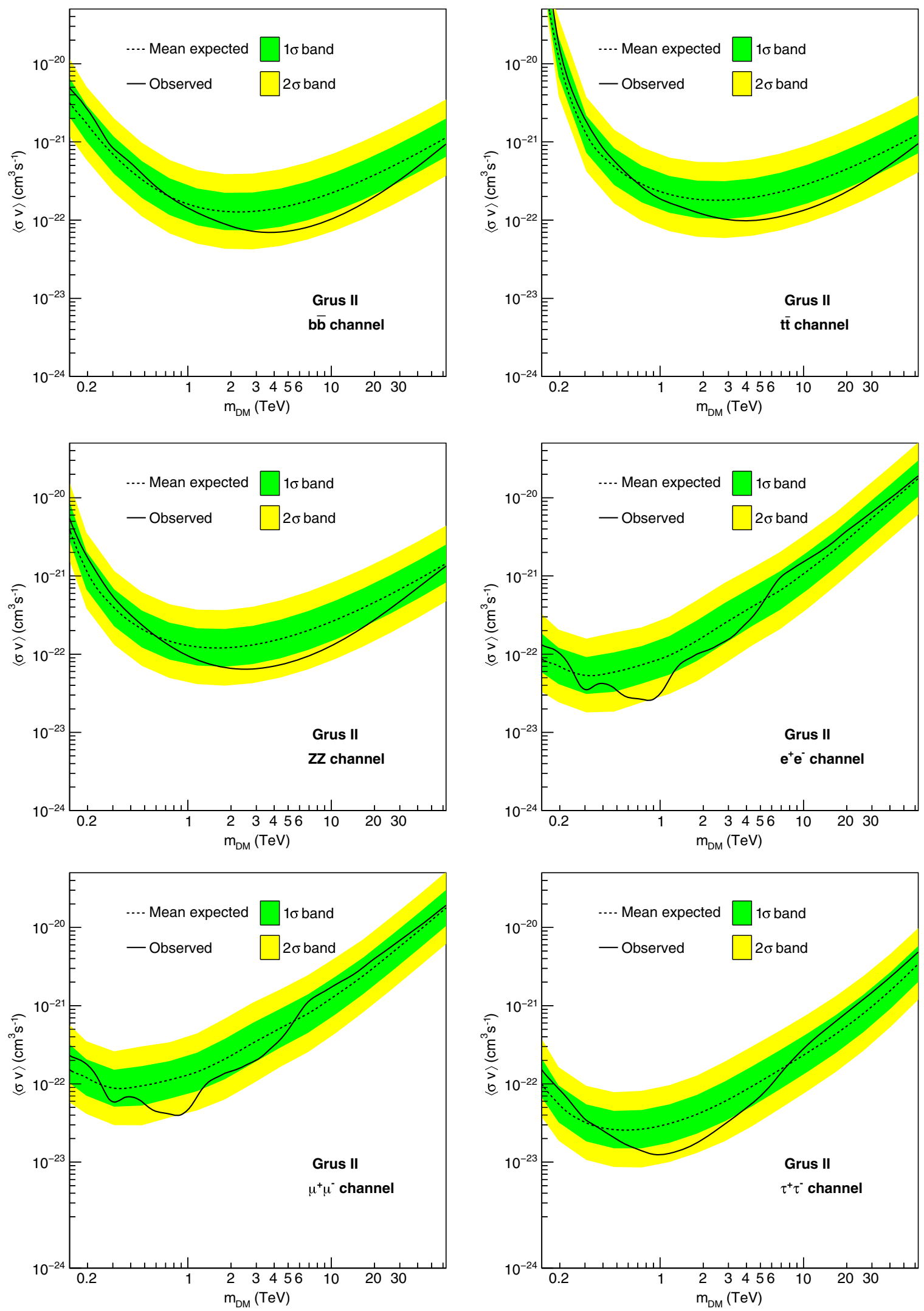

FIG. 12. 95\% C.L. upper limits on the annihilation cross section $\langle\sigma v\rangle$ for Gru II in the $b \bar{b}, t \bar{t}, Z Z, e^{+} e^{-}, \mu^{+} \mu^{-}, \tau^{+} \tau^{-}$annihilation channels, respectively, without the uncertainty on the $J$-factor. Observed limits (solid lines) together with mean expected limits (dashed line) and the $1 \sigma$ (green area) and $2 \sigma$ (yellow area) containment bands are shown, respectively. 
[1] R. Adam et al. (Planck Collaboration), Astron. Astrophys. 594, A1 (2016).

[2] G. Bertone, D. Hooper, and J. Silk, Phys. Rep. 405, 279 (2005).

[3] G. Steigman, B. Dasgupta, and J. F. Beacom, Phys. Rev. D 86, 023506 (2012).

[4] A. Viana, M. Medina, J. Penarrubia, P. Brun, J. Glicenstein, K. Kosack, E. Moulin, M. Naumann-Godo, and B. Peyaud, Astrophys. J. 746, 77 (2012).

[5] M. Winter, G. Zaharijas, K. Bechtol, and J. Vandenbroucke, Astrophys. J. Lett. 832, L6 (2016).

[6] K. N. Abazajian et al. (SDSS Collaboration), Astrophys. J. Suppl. Ser. 182, 543 (2009).

[7] http://pan-starrs.ifa.hawaii.edu/ (2008).

[8] http://www.darkenergysurvey.org/ (2008).

[9] http://www.lsst.org/lsst/ (2008).

[10] J. Binney and S. Tremaine, Galactic Dynamics: Second Edition (Princeton University Press, Princeton, NJ, 2008).

[11] A. Geringer-Sameth, S. M. Koushiappas, and M. Walker, Astrophys. J. 801, 74 (2015).

[12] K. Bechtol et al. (DES Collaboration), Astrophys. J. 807, 50 (2015).

[13] S. Li, Y.-F. Liang, K.-K. Duan, Z.-Q. Shen, X. Huang, X. Li, Y.-Z. Fan, N.-H. Liao, L. Feng, and J. Chang, Phys. Rev. D 93, 043518 (2016).

[14] S. E. Koposov, V. Belokurov, G. Torrealba, and N. Wyn Evans, Astrophys. J. 805, 130 (2015).

[15] V. Belokurov et al., Astrophys. J. 654, 897 (2007).

[16] A. Hamanowicz et al., Acta Astron. 66, 197 (2016).

[17] V. Bonnivard, C. Combet, D. Maurin, A. Geringer-Sameth, S. M. Koushiappas, M. G. Walker, M. Mateo, E. W. Olszewski, and J. I. Bailey III, Astrophys. J. 808, L36 (2015).

[18] A. Achterberg, M. van Beekveld, W. Beenakker, S. Caron, and L. Hendriks, J. Cosmol. Astropart. Phys. 12 (2015) 013.

[19] N. W. Evans, J. L. Sanders, and A. Geringer-Sameth, Phys. Rev. D 93, 103512 (2016).

[20] V. Bonnivard, D. Maurin, and M. G. Walker, Mon. Not. R. Astron. Soc. 462, 223 (2016).

[21] M. G. Walker, Mario Mateo, Edward W. Olszewski, John I. Bailey III, Sergey E. Koposov, Vasily Belokurov, and N. Wyn Evans, Astrophys. J. 808, 108 (2015).

[22] Q. E. Minor, A. B. Pace, J. L. Marshall, and L. E. Strigari, Mon. Not. R. Astron. Soc. 487, 2961 (2019).

[23] B. Mutlu-Pakdil, D. J. Sand, J. L. Carlin, K. Spekkens, N. Caldwell, D. Crnojević, A. K. Hughes, B. Willman, and D. Zaritsky, Astrophys. J. 863, 25 (2018).

[24] M. G. Walker et al., Astrophys. J. 819, 53 (2016).

[25] J. D. Simon et al. (DES Collaboration), Astrophys. J. 838, 11 (2017).

[26] A. Albert et al. (DES and Fermi-LAT Collaborations), Astrophys. J. 834, 110 (2017).

[27] A. B. Pace and L. E. Strigari, Mon. Not. R. Astron. Soc. 482, 3480 (2019).

[28] V. Bonnivard, C. Combet, D. Maurin, and M. G. Walker, Mon. Not. R. Astron. Soc. 446, 3002 (2015).

[29] V. Lefranc, G. A. Mamon, and P. Panci, J. Cosmol. Astropart. Phys. 09 (2016) 021.
[30] F. Aharonian et al. (H.E.S.S. Collaboration), Astron. Astrophys. 457, 899 (2006).

[31] M. de Naurois and L. Rolland, Astropart. Phys. 32, 231 (2009).

[32] R. D. Parsons and J. A. Hinton, Astropart. Phys. 56, 26 (2014).

[33] M. Holler et al. (H.E.S.S. Collaboration), Proc. Sci. ICRC2015 (2016) 980 [arXiv:1509.02896].

[34] D. Berge, S. Funk, and J. Hinton, Astron. Astrophys. 466, 1219 (2007).

[35] T.-P. Li and Y.-Q. Ma, Astrophys. J. 272, 317 (1983).

[36] M. Cirelli, G. Corcella, A. Hektor, G. Hütsi, M. Kadastik, P. Panci, M. Raidal, F. Sala, and A. Strumia, J. Cosmol. Astropart. Phys. 03 (2011) 051.

[37] G. Cowan, C. K., E. Gross, and O. Vitells, Eur. Phys. J. C 71, 1554 (2011).

[38] A. Abramowski et al. (H.E.S.S. Collaboration), Phys. Rev. D 90, 112012 (2014).

[39] V. Bonnivard et al., Mon. Not. R. Astron. Soc. 453, 849 (2015).

[40] J. Aleksic et al. (MAGIC Collaboration), J. Cosmol. Astropart. Phys. 02 (2014) 008.

[41] M. L. Ahnen et al. (MAGIC and Fermi-LAT Collaborations), J. Cosmol. Astropart. Phys. 02 (2016) 039.

[42] S. Archambault et al. (VERITAS Collaboration), Phys. Rev. D 95, 082001 (2017).

[43] M. Ackermann et al. (Fermi-LAT Collaboration), Phys. Rev. Lett. 115, 231301 (2015).

[44] A. Albert et al. (HAWC Collaboration), Astrophys. J. 853, 154 (2018).

[45] E. N. Kirby, J. G. Cohen, J. D. Simon, P. Guhathakurta, A. O. Thygesen, and G. E. Duggan, Astrophys. J. 838, 83 (2017).

[46] H. Abdalla et al. (H.E.S.S. Collaboration), J. Cosmol. Astropart. Phys. 11 (2018) 037.

[47] M. Ackermann et al. (Fermi-LAT Collaboration), Phys. Rev. D 91, 122002 (2015).

[48] V. Bonnivard, D. Maurin, and M. G. Walker, Mon. Not. R. Astron. Soc. 462, 223 (2016).

[49] A. Ibarra, A. S. Lamperstorfer, S. López-Gehler, M. Pato, and G. Bertone, J. Cosmol. Astropart. Phys. 09 (2015) 048; 06 (2016) E02.

[50] F. Giacchino, A. Ibarra, L. Lopez Honorez, M. H. G. Tytgat, and S. Wild, J. Cosmol. Astropart. Phys. 02 (2016) 002.

[51] M. Duerr, P. Fileviez Perez, and J. Smirnov, Phys. Rev. D 92, 083521 (2015).

[52] M. Baumgart, T. Cohen, I. Moult, N. L. Rodd, T. R. Slatyer, M. P. Solon, I. W. Stewart, and V. Vaidya, J. High Energy Phys. 03 (2018) 117.

[53] M. Baumgart, T. Cohen, E. Moulin, I. Moult, L. Rinchiuso, N. L. Rodd, T. R. Slatyer, I. W. Stewart, and V. Vaidya, J. High Energy Phys. 01 (2019) 036.

[54] R. Mahbubani and L. Senatore, Phys. Rev. D 73, 043510 (2006).

[55] J. Kearney, N. Orlofsky, and A. Pierce, Phys. Rev. D 95, 035020 (2017).

[56] K. Kowalska and E. M. Sessolo, Adv. High Energy Phys. 2018, 6828560 (2018). 